\title{
ARTICLE
}

\section{A dendritic cell-based systemic vaccine induces long-lived lung- resident memory Th17 cells and ameliorates pulmonary mycosis}

Keigo Ueno ${ }^{1}$, Makoto Urai ${ }^{1,5}$, Sota Sadamoto ${ }^{2}$, Minoru Shinozaki ${ }^{2}$, Shogo Takatsuka ${ }^{1}$, Masahiro Abe ${ }^{1}$, Yoshiko Otani ${ }^{1,3}$, Nao Yanagihara ${ }^{1,3}$, Kiminori Shimizu $^{3}$, Yoichiro Iwakura ${ }^{4}$, Kazutoshi Shibuya ${ }^{2}$, Yoshitsugu Miyazaki ${ }^{1}$ and Yuki Kinjo ${ }^{1,6}$

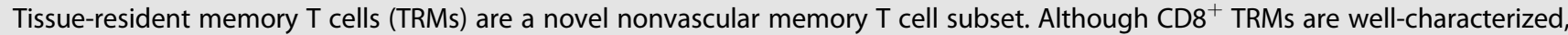
$\mathrm{CD}^{+}{ }^{+}$TRMs-especially lung-resident memory Th17 cells-are still being defined. In this study, we characterized lung-resident memory Th17 cells (lung TRM17) and their role in protection against the highly virulent fungus Cryptococcus gattii. We found that intravenously transferred DCs preferentially migrated to lungs and attracted recipient DCs and led to the induction of long-lived Th17 cells expressing characteristic markers. This population could be clearly discriminated from circulating T cells by intravascular staining and was not depleted by the immunosuppressive agent FTY720. The $C$. gattii antigen re-stimulation assay revealed that vaccine-induced lung Th17 cells produced IL-17A but not IFNy. The DC vaccine significantly increased IL-17A production and suppressed fungal burden in the lungs and improved the survival of mice infected with $C$. gattii. This protective effect was significantly reduced in the IL-17A knockout (KO) mice, but not in the FTY720-treated mice. The protective effect also coincided with the activation of neutrophils and multinucleated giant cells, and these inflammatory responses were suppressed in the vaccinated IL-17A KO mice. Overall, these data demonstrated that the systemic DC vaccine induced lung TRM17, which played a substantial role in anti-fungal immunity.

Mucosal Immunology (2019) 12:265-276; https://doi.org/10.1038/s41385-018-0094-4

\section{INTRODUCTION}

Tissue-resident memory T cells (TRMs) are a novel subpopulation of memory $T$ cells residing in a variety of non-lymphoid peripheral tissues including the gut, lung, skin, genital tract, salivary gland, liver, and the brain ${ }^{1}$. Because they are typically located at barrier frontlines, they are thought to be involved in protection against the pathogens including viruses, bacteria, and parasites. However, several types of TRMs, including Th2-type TRMs, appear to participate in inflammatory diseases including asthma and colitis ${ }^{2}$. Therefore, modulating the behavior of TRMs is potentially important in controlling infections and inflammatory diseases. Similar to TRMs, tumor-infiltrating lymphocytes (TILs) also reside in tumor tissues, and cytotoxic T lymphocytes (CTLs)-and related TILs have the ability to improve the prognosis. Adoptive transfer of TILs is also expected to suppress growing tumors ${ }^{3}$.

TRMs can be discriminated from circulating lymphocytes and characteristically express CD69, a receptor of myosin light chain 9 (Myl9). Myl9 is secreted from platelets concomitantly with local inflammation, and forms a net-like structure (Myl9 net) on the endothelial surface. CD69-expressing activated lymphocytes can become anchored in blood vessels in a CD69- and Myl9dependent manner and infiltrate tissues ${ }^{4}$. Further, expression of CD69 can downregulate the sphingosine-1-phosphate receptor (S1PR), which is essential for transport across blood vessel walls ${ }^{5}$. Similar to TRMs, TILs also express TRM-like markers, including CD69 and CD103 (aEß7 integrin) $)^{3}$.

$\mathrm{CD}^{+}$TRMs are well-characterized, but less is known about $\mathrm{CD}^{+}$TRMs, especially Th17-type TRMs in the lungs. A memory Th17 cell-inducing vaccine is especially awaited for respiratory infections including tuberculosis, pneumococcal infection, pertussis, and flu ${ }^{6}$. Therefore, lung-resident memory Th17 cells could be an important target and a key player for vaccine development.

In a previous study, we developed a dendritic cell-based vaccine (DC vaccine) against life-threatening pulmonary cryptococcosis caused by the highly virulent pathogenic fungus Cryptococcus gattii ${ }^{7}$. An outbreak of C. gattii infection occurred in North America during 1999, and cryptococcosis caused by C. gattii is perceived as an emerging infectious disease. The highly virulent $C$. gattii can infect healthy individuals, and the mortality rate has been $8-20 \% 8$. Public health authorities in British Columbia and the USA have

\footnotetext{
${ }^{1}$ Department of Chemotherapy and Mycoses, National Institute of Infectious Diseases, 1-23-1 Toyama, Shinjuku-ku, Tokyo 162-8640, Japan; ${ }^{2}$ Department of Surgical Pathology, Toho University School of Medicine, 6-11-1 Omori-Nishi, Ota-ku, Tokyo 143-8541, Japan; ${ }^{3}$ Department of Biological Science and Technology, Faculty of Industrial Science and Technology, Tokyo University of Science, 6-3-1 Niijuku, Katsushika-ku, Tokyo 125-8585, Japan and ${ }^{4}$ Division of Experimental Animal Immunology, Research Institute for Biological Sciences, Tokyo University of Science, 2669 Yamazaki, Noda, Chiba 278-0022, Japan

Correspondence: Keigo Ueno (keigo-u@niid.go.jp) or Yuki Kinjo (ykinjo@niid.go.jp)

${ }^{5}$ Present address: Department of Chemistry for Life Sciences and Agriculture, Faculty of Life Sciences, Tokyo University of Agriculture, 1-1-1 Sakuragaoka, Setagaya-ku, Tokyo 1568502, Japan

${ }^{6}$ Present address: Department of Bacteriology, The Jikei University School of Medicine, 3-25-8 Nishi-Shimbashi, Minato-ku, Tokyo 105-8461, Japan
}

Received: 1 December 2017 Revised: 14 September 2018 Accepted: 17 September 2018

Published online: 2 October 2018 
brought this situation to the public's attention'. However, a clinical vaccine against cryptococcosis has not yet been developed, and protective memory against $C$. gattii infection has not been demonstrated.

DC vaccines are widely utilized in cancer therapy ${ }^{10}$ and infection control ${ }^{11}$, as well as in basic research to analyze T-cell response $^{12}$. The DC vaccine that we have developed comprises mouse bone marrow-derived DCs pulsed with heat-inactivated acapsular C. gattii cells, in which the capsule synthesis-related gene CAP60 has been disrupted using a reverse genetics approach. When the DC vaccine was intravenously transferred into a murine host, we observed induction of cytokine-producing $T$ cells in the lungs and amelioration of pulmonary cryptococcosis caused by $C$. gattii ${ }^{7}$. However, induction of memory T cells and any long-term protective effect conferred by the DC vaccine remained to be elucidated.

In the current study, we investigated memory T cells induced by the $D C$ vaccine that contributed to its long-term protective effect. We also evaluated the migration of transferred DCs, distribution of C. gattii-specific memory $\mathrm{T}$ cells, and longevity and protective effect of the memory T cells. We demonstrate that the systemic DC vaccine induced lung-resident memory Th17 cells, which participated in protective inflammatory responses including neutrophil activation and granuloma formation in pulmonary cryptococcosis caused by a highly virulent $C$. gattii strain.

\section{RESULTS}

Antigen-pulsed DCs preferentially accumulated in the lungs after intravenous transfer

Initially, we evaluated migration of transferred DCs and activation of recipient DCs. Donor DCs were prepared from C57BL/6 (CD45.1) mice, and then intravenously transferred into recipient $\mathrm{C} 57 \mathrm{BL} / 6$ (CD45.2) mice (Fig. 1a). After $18 \mathrm{~h}$ following the transfer, the antigen-pulsed DCs preferentially accumulated in the lungs, compared with spleen and mediastinal lymph node (MedLN) (Fig. 1b). Recipient DCs (CD45.2 $2^{+}$Ly6G low Siglec-F $^{\text {low }} \mathrm{CD} 11 \mathrm{c}^{+} \mathrm{I}-\mathrm{Ab}^{+}$ cells) significantly increased in the lungs of the mice that had received the antigen-pulsed DCs, but not in their spleen and MedLN (Fig. 1c), suggesting that the antigen-pulsed DC migrates to the lungs after the intravenous transfer and attracts recipient DCs.

The systemic DC vaccine induced lung-resident memory Th17 cells (lung TRM17)

Next, we evaluated memory $T$ cells in the lungs, spleen, and bone marrow (BM) after intravenous immunization with the antigen-pulsed DCs (Fig. 2a). Memory T cells were categorized as previously reported ${ }^{13}$. More than 14 weeks after the final immunization, $\mathrm{CD}^{+}{ }^{+} \mathrm{T}$ cells expressing the TRM-marker CD69 were significantly increased in the lungs of the immunized mice. In contrast, this cell population was not increased in the spleen and BM of the immunized mice (Fig. 2a). Further, the DC vaccine also induced $\mathrm{CD}^{+}$T-cells expressing tissue-resident markers in the lungs, but to a lesser extent than their CD4 ${ }^{+}$ counterparts (Supplementary Figure S1A). Vaccine-induced $\mathrm{CD}^{+}{ }^{+} \mathrm{T}$ cells expressing TRM-profiles were detected in the lungs for at least 6 months after vaccination (Supplementary Figure S2). These results suggest that the DC vaccine induces long-lived $\mathrm{CD}^{+}$TRMs in the lungs, despite intravenous administration.

In general, TRMs immediately produced cytokines after antigen re-stimulation ${ }^{1,13}$. Therefore, we evaluated cytokine production of vaccine-induced $T$ cells after polyclonal re-stimulation with phorbol 12-myristate 13-acetate (PMA) and ionomycin (Fig. 2b). IL-17A-producing $\mathrm{CD}^{+} \mathrm{T}$ cells significantly increased in the lungs $>10$ weeks after the final vaccination (Fig. 2b). This increase was clearly higher than that in the spleen and BM, and that in lung

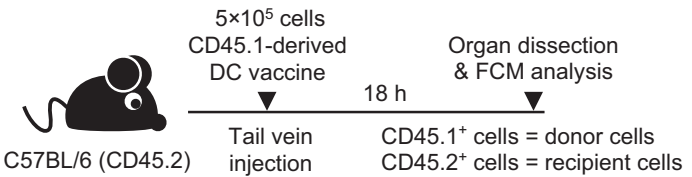

b Parent gate:

Ly6Glow, Siglec-Flow cells

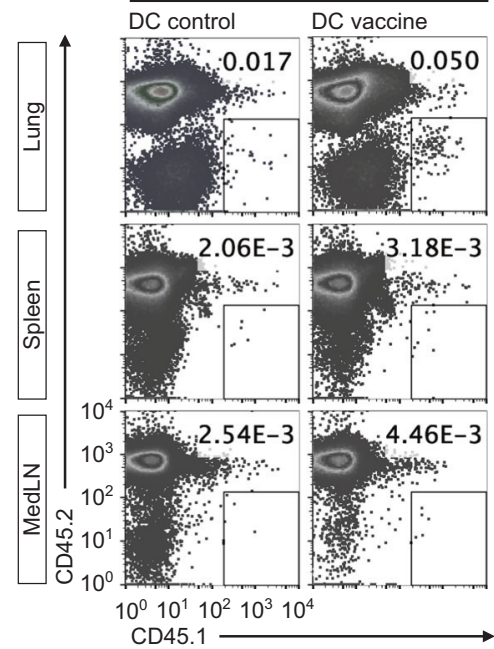

$\square$ DC control

DC vaccine

CD45.1+ donor cells (\% of parent)

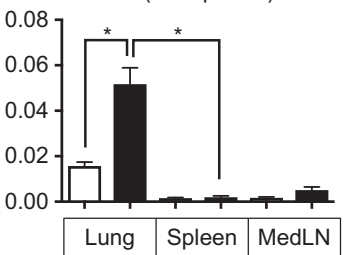

CD45.1+ donor cells (cells/organ)

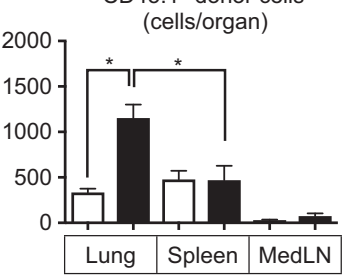

C

Parent gate:

Ly6G/siglec-Flow, CD45.2 ${ }^{\text {high }}$ cells

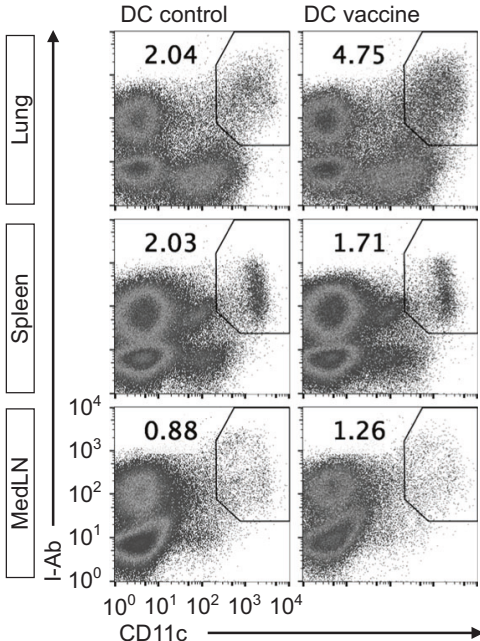

$\square \mathrm{DC}$ control $\square \mathrm{DC}$ vaccine CD45.2 $2^{+}$recipient-DCs (\% of parent)

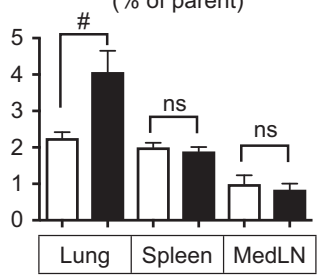

CD45. $2^{+}$recipient-DCs $\left(\times 10^{4}\right.$ cells/organ)

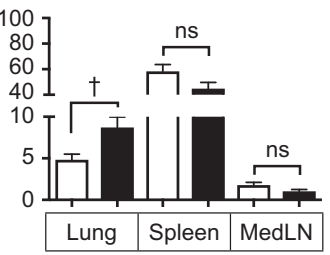

Fig. 1 Antigen-pulsed DCs migrated to the lungs, and attracted recipient DCs after intravenous transfer. a Experiment design. Antigen-pulsed or unpulsed DCs $\left(5 \times 10^{5}\right.$ cells/mouse) were derived from C57BL/6 (CD45.1) mice. C57BL/6 (CD45.2) mice were used as recipients, and received $5 \times 10^{5}$ donor cells (CD45.1) via tail vein. The lungs, spleen, and mediastinal lymph node (MedLN) were dissected after $18 \mathrm{~h}$ of the transfer to measure donor and recipient cells. b Donor cells were preferentially detected in the lungs after transfer. For flow cytometry analysis, gates were set for Ly6G low Siglec-F ${ }^{\text {low }}$ cells. c Recipient DCs were increased in the lungs of the immunized mice. For flow cytometry analysis, gates were set for Ly6G ${ }^{\text {low }}$ Siglec$\mathrm{F}^{\text {low }}, \mathrm{CD} 45.2^{+}$cells. Representative flow cytometry profiles (left) and bar graphs for statistical analysis (right) are shown in each panel. Pooled data from two independent experiments were used to prepare bar graphs (means \pm SEMs,$n=6$ ). ${ }^{*} P<0.05$ in analysis of variance with Tukey's post hoc test; ${ }^{\#} P<0.05$ in unpaired $t$-test with Welch's correction; ${ }^{+} P<0.05$ in unpaired $t$-test; ns no significant difference 
a

a

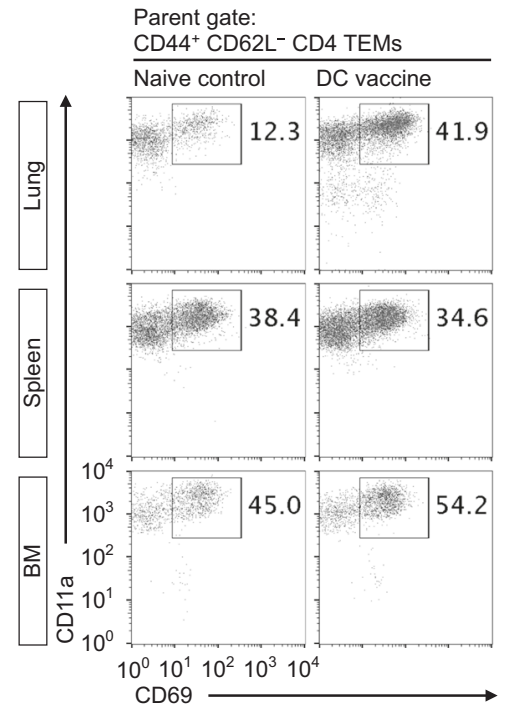

$\square$ Naive control

CD11a ${ }^{+} \mathrm{CD}^{\circ}{ }^{+} \mathrm{CD} 4$ TRMs ( $\%$ of parent)

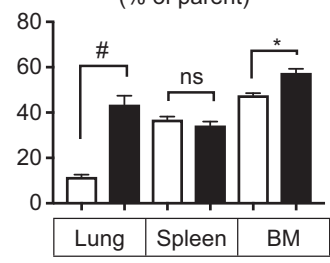

$\mathrm{CD}_{11 \mathrm{a}^{+} \mathrm{CD}^{2} 9^{+} \mathrm{CD} 4 \mathrm{TRMs}}$ (Log cells/organ)

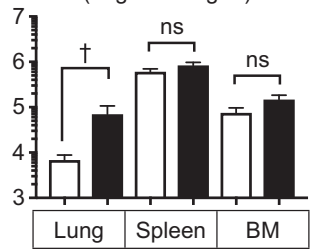

b

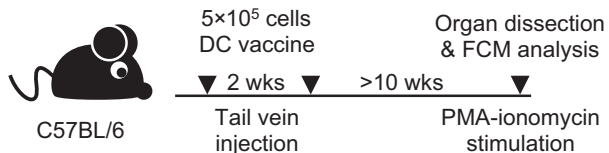

Parent gate: CD44+ CD62L- CD4 T cells

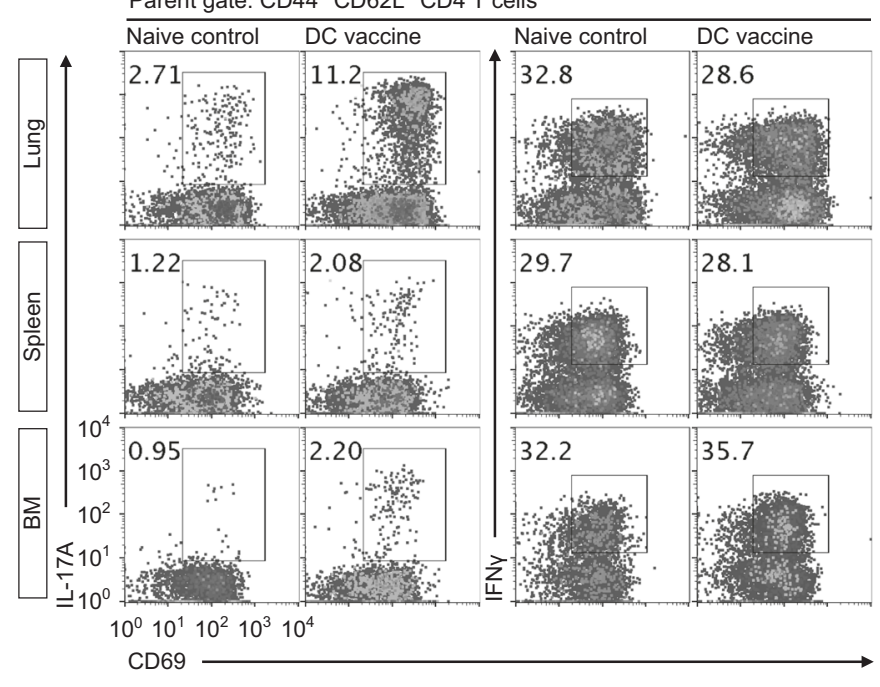

$\square$ Naive control $\square$ DC vaccine

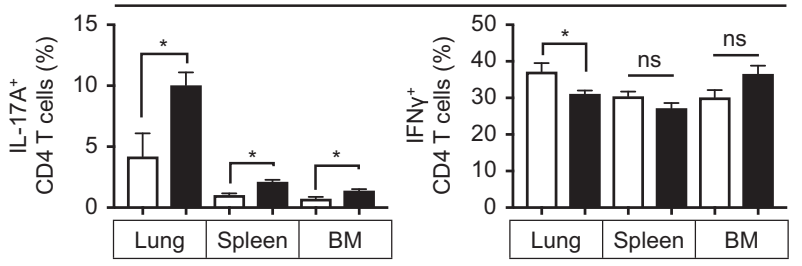

C
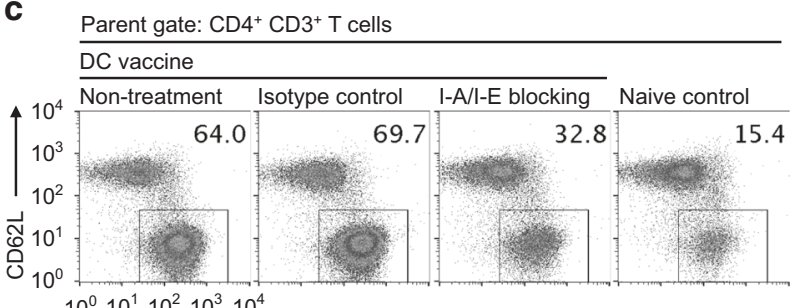

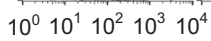

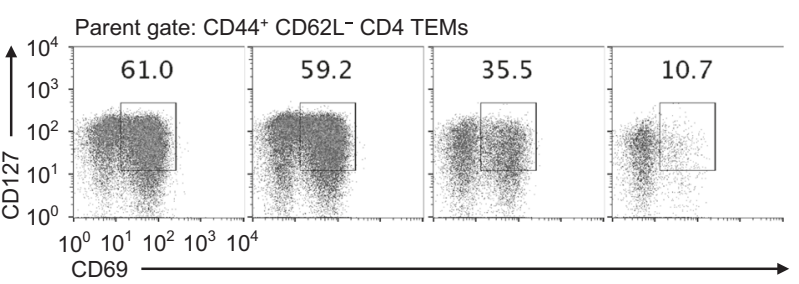

CD69
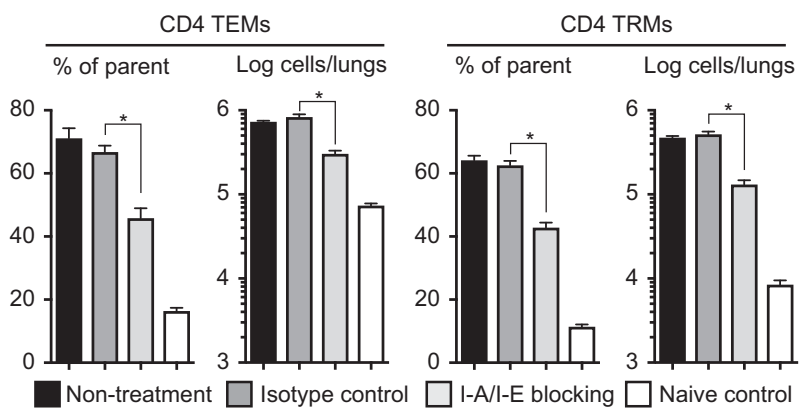

IL-17A ${ }^{+}$CD8 ${ }^{+}$T-cells (Supplementary Figure S1B). However, the vaccine did not induce IFNy-producing $T$ cells (Fig. $2 b$ and Supplementary Figure S1B). These results suggest that the vaccine induces Th17-type TRMs in the lungs.
Next, we investigated how donor DCs were responsible for the generation of lung $\mathrm{CD}^{+}{ }^{+}$TRMs (Fig. 2c). In this experiment, we utilized antigen-pulsed DCs pretreated by the major histocompatibility complex class-II (MHC-II) blocking antibody (M5/114.15.2, 
Fig. 2 The DC vaccine induced lung $\mathrm{CD}^{+} \mathrm{T}$ cells expressing memory and tissue-resident markers. a Number of lung CD4 ${ }^{+} \mathrm{T}$ cells increased in the lungs of the immunized mice. Antigen-pulsed DCs were injected twice every 2 weeks via the tail vein. The lungs, spleen, and BM were dissected $>14$ weeks after transfer to evaluate memory $T$ cells. For flow cytometry analysis, gates were set for the effector memory CD4 ${ }^{+}$

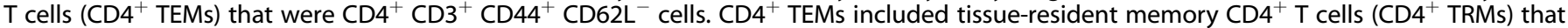

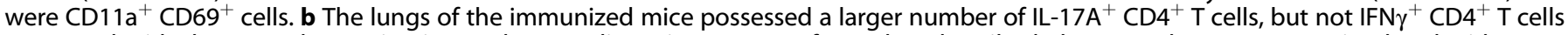
compared with the controls. Vaccination and organ dissection were performed as described above. Leukocytes were stimulated with PMAionomycin for $4 \mathrm{~h}$. Brefeldin A and monensin were added for the final $2 \mathrm{~h}$ of culture to stain the intracellular cytokines. c MHC-II on transferred donor DC was partially required for the development of lung $C D 4^{+}$TRMs. Prior to the transfer, antigen-pulsed DCs were treated with $10 \mu \mathrm{g} / \mathrm{mL}$ anti-I-A/I-E mAb (M5/114.15.2) to block surface MHC-II on DCs. DCs were then washed twice with phosphate buffered saline (PBS). Vaccination was performed as described above, and lung leukocytes were harvested 6 weeks after the transfer to evaluate the development of CD4 ${ }^{+}$TEMs and $\mathrm{CD} 127^{+} \mathrm{CD}_{69}{ }^{+}$TRM. Representative flow cytometry profiles and bar graphs for statistical analysis are shown in each panel. Pooled data from more than two independent experiments were used to prepare bar graphs [means $\pm \mathrm{SEMs}, \mathbf{a} n=7, \mathbf{b} n=6-9$, and $\mathbf{c} n=6$ ]. ${ }^{*} P<0.05$ in unpaired $t$-test; ${ }^{\#} P<0.05$ in unpaired $t$-test with Welch's correction; ${ }^{\dagger} P<0.05$ in Mann-Whitney $U$ test; ns no significant difference

may also be referred to as M5/114 in the literature). This blocking antibody has been previously used in in vivo studies ${ }^{14,15}$. This blocking experiment revealed that the number of lung $\mathrm{CD}^{+}{ }^{+}$TEMs and TRMs significantly decreased in the mice that received the MHC-II-blocked DC vaccine; however, the MHC-II-blocked DC vaccine still had the ability to induce $\mathrm{CD}^{+}$TRMs in the lungs (Fig. 2C), suggesting that MHC-II on donor DCs is partially required for the generation of lung CD4 ${ }^{+}$TRMs, and that the attracted recipient DCs are also involved in the development of lung $C D 4^{+}$ TRMs.

Because it has been shown that TRMs are not depleted by the administration of the drug FTY720 (fingolimod) and not stained by intravascular (IV) staining ${ }^{16}$, we tested the effect of FTY720 administration and IV staining in $\mathrm{CD}^{+}$TRMs. We confirmed that vascular $\mathrm{CD}^{+}{ }^{+} \mathrm{T}$ cells were clearly depleted by FTY720 treatment in this model and were completely stained by IV staining (Fig. 3a). Similarly, vaccine-induced lung $\mathrm{CD} 69^{+} \mathrm{CD} 4^{+}$TRMs were also not depleted by FTY720 and not labeled by IV staining, and the number of $\mathrm{CD}_{45 \mathrm{iv}^{-}} \mathrm{CD} 9^{+} \mathrm{CD}^{+}{ }^{+}$TRMs (approximately $6 \times 10^{4}$ cells/lung) was still significantly greater than that in the counterpart of non-vaccinated controls after the FTY720 treatment (Fig. 3b). We also found that this population constitutively expressed the Th17-defining transcriptional factor RORyt but not T-bet (Fig. $3 \mathrm{~b}$ and Supplementary Figure S3A). The vaccineinduced lung $\mathrm{CD}^{+}{ }^{+}$TRMs also expressed Ki-67, CD127, CXCR3, TCF-7, Sca1, and CD43 (Supplementary Figures S3B and S3C). Histological analysis revealed that vaccine-induced $\mathrm{CD}^{+}{ }^{+}$TRMs localized in the narrow space around airways and near blood vessels (Fig. 3c), which is consistent with the localization of fluinduced lung CD4 ${ }^{+}$TRMs $^{17}$. These results also suggest that the DC vaccine induces lung $\mathrm{CD}^{+}{ }^{+}$TRMs.

We also investigated antigen-specific memory of $\mathrm{CD}^{+}$TRMs against $C$. gattii (Fig. 4a, b). After FTY720 treatment to deplete vascular lymphocytes as described in Fig. $3, \mathrm{CD}^{+} \mathrm{T}$ cells were stimulated by antigen-pulsed BMDCs (Fig. 4a). Vaccine-induced lung $\mathrm{CD}^{+}{ }^{+}$TRMs produced IL-17A, but not IFNy after in vitro restimulation, and vaccine-induced spleen $\mathrm{CD}^{+}{ }^{+} \mathrm{T}$ cells also produced IL-17A after re-stimulation, but its population ratio was lesser than that in the lung counterparts (Fig. 4a). Because we used the heat-inactivated acapsular C. gattii strain CAP60 $\triangle$ as an antigen to stimulate $\mathrm{CD} 4^{+}$T cells in this experiment (Fig. $4 \mathrm{a}$ ), we next investigated whether the capsular $C$. gattii strain and nonrelated pathogenic fungus Candida albicans also stimulated vaccine-induced memory $T$ cells (Fig. 4b). The heat-inactivated capsular C. gattii strain R265 also induced immunized lungs, but not immunized spleen, to secrete IL-17A production; however, heat-inactivated C. albicans SC5314 did not induce IL-17A secretion (Fig. 4b). These results suggest that vaccine-induced lung $\mathrm{CD}^{+}{ }^{+}$TRMs are memory Th17 cells that respond to $C$. gattiiderived antigens.

Taken together, these results indicate that the systemic DC vaccine induces lung-resident memory Th17 cells, and that these cells are maintained for at least 6 months after immunization. Hereafter, we refer to this population as lung TRM17.

\section{IL-17A was required for the generation and maintenance of} vaccine-induced lung TRM17

A previous study showed that lung $\mathrm{CD}_{103^{+}} \mathrm{CD}^{+}$TRMs were helped by $\mathrm{CD}^{+}{ }^{+} \mathrm{T}$ cells in an IFNy-dependent manner ${ }^{18}$, but the key cytokine for generation or maintenance of lung $\mathrm{CD}^{+}{ }^{+}$TRMs remained to be elucidated. We showed that vaccine-induced lung TRM17 could produce IL-17A without antigen re-stimulation and that they expressed the IL-17A receptor IL-17RA (Figs. 4 and 5a, b). These features of lung TRM17 have raised the possibility that IL$17 \mathrm{~A}$ is a key factor for the generation or maintenance of lung TRM17. Thus, we measured the amount of lung TRM17 in the wildtype (WT) C57BL/6 and IL-17A-knockout (KO) mice 2 or 15 weeks after the final vaccination (Fig. $5 \mathrm{c}$ ). At the primary time point, lung TRM17 modestly increased in the IL-17A-KO mice that had received the DC vaccine; however, the amount of lung TRM17 in the IL-17A KO mice was significantly lower than that in the WT mice. At the memory time point, the amount of lung TRM17 in the IL-17A KO mice decreased to non-vaccinated baseline, and CD127 expression on lung $\mathrm{CD} 4^{+}$TEMs was also significantly attenuated in them (Fig. 5C). These results suggest that IL-17A is involved in both the development and maintenance of vaccine-mediated lung TRM17 in this model.

Lung TRM17 was involved in the vaccine-mediated protective effect against highly virulent $C$. gattii infection

Our previous study showed that the DC vaccine induced protective immunity against highly virulent $C$. gattii when infected soon after the last immunization ${ }^{7}$. However, the long-term protective effect was not evaluated. Therefore, we tested the long-term protective effect of the DC vaccine in the murine pulmonary infection model. The infection challenge was performed after $>2$ months from the last immunization (Fig. 6a). Fungal burden at day 14 post-infection was significantly lower in the lungs of the immunized mice, even if the infection challenge was performed after 6 months from the last immunization (Fig. 6a). Amounts of IL-17A and IFNy were significantly higher in the lungs of the immunized mice than in those of non-vaccinated controls (Fig. 6a). Furthermore, survival was also significantly improved in the immunized mice (Fig. $6 \mathrm{~b}$ ). These results suggest the long-term protective effect of the DC vaccine, and long-term protective effect appeared to be correlated to long-term retention of lung TRM17.

Next, we tested whether lung TRM17 contributed to the longterm protective effect of the DC vaccine. As described above, both the generation and maintenance of lung TRM17 were significantly reduced in the IL-17A KO mice (Fig. 5), and the effector function of lung TRM17 also appeared to be depressed in these mice. Indeed, in the IL-17A KO mice, the DC vaccine did not suppress fungal burden in the lungs at day 14 post-infection (Fig. 6c). Furthermore, 

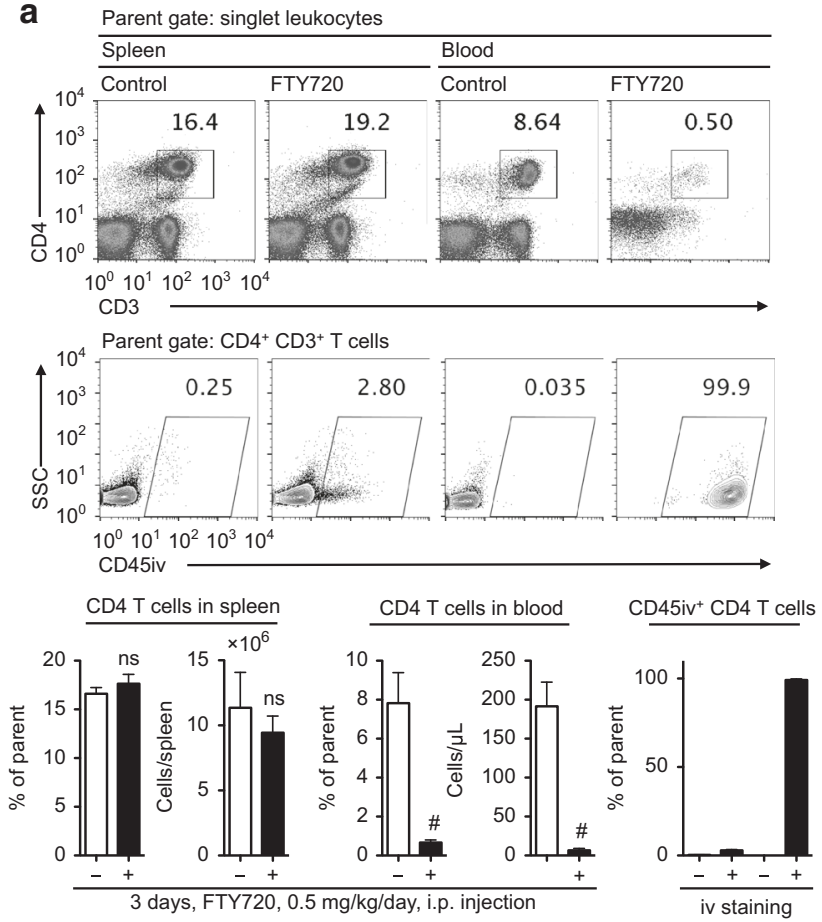

b Parent gate:
Lung CD44 ${ }^{+} \mathrm{CD}^{2} \mathrm{~L}^{-} \mathrm{CD} 4 \mathrm{TEMs}$
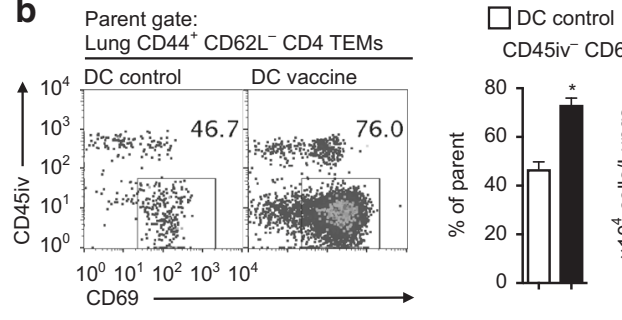
DC vaccine
$9^{+}$CD4 TRMs

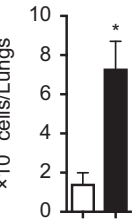

Parent gate:

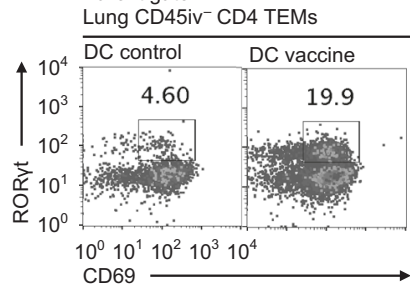

$\square \mathrm{DC}$ control

DC vaccine CD45iv ${ }^{-}$RORyt ${ }^{+}$CD4 TRMs

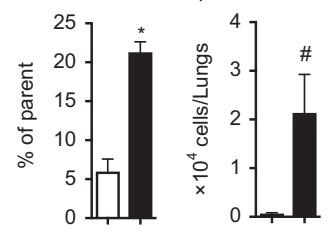

C

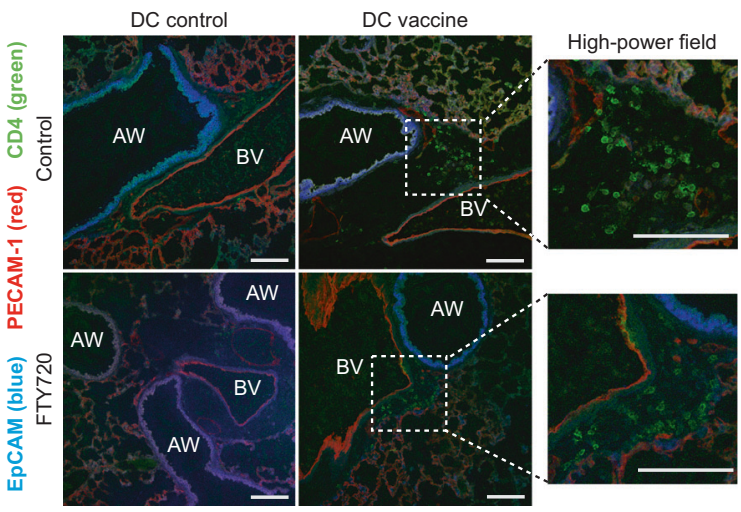

depletion of circulating lymphocytes did not disturb vaccinemediated fungal reduction in lungs (Fig. $6 \mathrm{~d}$, e), suggesting that vaccine-mediated protection depends on tissue-resident
Fig. 3 Evaluation of tissue-resident $\mathrm{CD}^{+}{ }^{+} \mathrm{T}$ cells with a combination of FTY720 treatment and IV staining. a Vascular CD4 ${ }^{+}$T cells were completely depleted by FTY720 treatment and stained by IV staining. Vaccination was performed as described above. The immune-suppressive agent, FTY720 (fingolimod, $0.5 \mathrm{mg} / \mathrm{kg} /$ day), was administrated into the peritoneum (i.p.) for 3 sequential days to deplete circulating lymphocytes 15 weeks after the final vaccination. On the following day, anti-CD45 mAb (FITC-labeled, $2.5 \mu \mathrm{g} /$ mouse) was injected into the orbital vein to stain the vascular leukocytes (IV staining) 5 min before euthanasia. Splenocytes and peripheral blood leukocytes were collected to validate the efficacy of FTY720 administration and IV staining. $\mathbf{b}$ The DC vaccine induced lung $\mathrm{CD}^{+}{ }^{+}$cells that were not depleted by FTY720 and were unlabeled by IV staining. To measure lung $\mathrm{CD}^{+} \mathrm{TRM}$, gates were set for $\mathrm{CD}^{+}{ }^{+}$TEMs and IV staining negative (CD45iv ${ }^{-}$) CD4 ${ }^{+}$TEMs for flow cytometry analysis. a, b Representative flow cytometry profiles and bar graphs for statistical analysis are shown. Pooled data from two independent experiments were used to prepare bar graphs (means \pm SEMs, $n=6$ ). ${ }^{*} P<0.05$ in unpaired $t$-test; ${ }^{*} P<0.05$ in unpaired $t$-test with Welch's correction; ns no significant difference. c Vaccine-induced $\mathrm{CD}^{+}$TRMs localized in the narrow space around airways and near blood vessels. Vaccination and FTY720administration were performed as described above, and frozen lung sections $(8 \mu \mathrm{m})$ were prepared 5 months after the final vaccination. Cryosections were fixed in fixation buffer (4\% PFA in PBS) and then stained with the antibodies depicted in the figure. The sections were observed under confocal laser microscopy. AW airways; BV blood vessels; bar $=100 \mu \mathrm{m}$

immunity. These results suggest that lung TRM17 contributes to the vaccine-mediated protective effect against highly virulent $C$. gattii.

Induction of neutrophils and granuloma depended on IL-17A in the lungs of the immunized mice after the infection challenge Next, we examined the role of vaccine-induced lung TRM17 after the infection challenge. In this analysis, we used WT and IL-17A KO mice and continuously administrated the drug FTY720 to deplete circulating lymphocytes during the challenge, as described in Fig. 6d, e. We analyzed leukocytes by flow cytometry at day 7 post-infection (Fig. 7) and by histological evaluation of lung sections at day 14 post-infection (Fig. 8). Gates for flow cytometry were set as previously described in a murine cryptococcosis model $^{19}$ and an asthma model ${ }^{20}$. At day 7 post-infection, lung leukocytes-including neutrophils, inflammatory monocytes/dendritic cells, and $\mathrm{CD} 4^{+}$TRMs - were significantly increased in the WT mice that had received the DC vaccine. However, the vaccine did not increase lung neutrophils in the IL-17A KO mice. Furthermore, the vaccine induced neutrophils to express Siglec- $\mathrm{F}$ and CD11C in an IL-17A-dependent manner (Fig. 7a). Because populations of Siglec- $\mathrm{F}^{\text {high }}$ Ly6G ${ }^{\text {low }}$ SSC high eosinophils and alveolar macrophages were detected outside of the neutrophilgate, neutrophils expressing Siglec-F and CD11c were clearly distinguishable from other cells. Previous studies have shown that a subpopulation of activated neutrophils expressed Siglec-F and CD11c on the cell surface ${ }^{21-25}$. Siglec- $F$ is known to be an inhibitory receptor that can be expressed in activated lung $T$ cells as well as eosinophils ${ }^{26}$. Although vascular $\mathrm{CD}^{+} \mathrm{T}$ cells were significantly depleted in this model as shown in Fig. 7c, the number of $\mathrm{CD} 127^{+} \mathrm{CD} 4^{+}$TRMs significantly increased in the lungs of the WT and IL-17A-KO mice that had received the DC vaccine, compared with before infection (Fig. 7b). Because CD69 expression significantly increased in the immunized mice, compared with the non-immunized mice, $\mathrm{CD}_{127^{+}} \mathrm{CD}^{+}$TRMs might be activated after the infection challenge (Fig. 7b); however, the effector function of $\mathrm{CD}_{127^{+}} \mathrm{CD}^{+}$TRMs appeared to be depressed in the IL-17A KO mice. These results suggest that the 

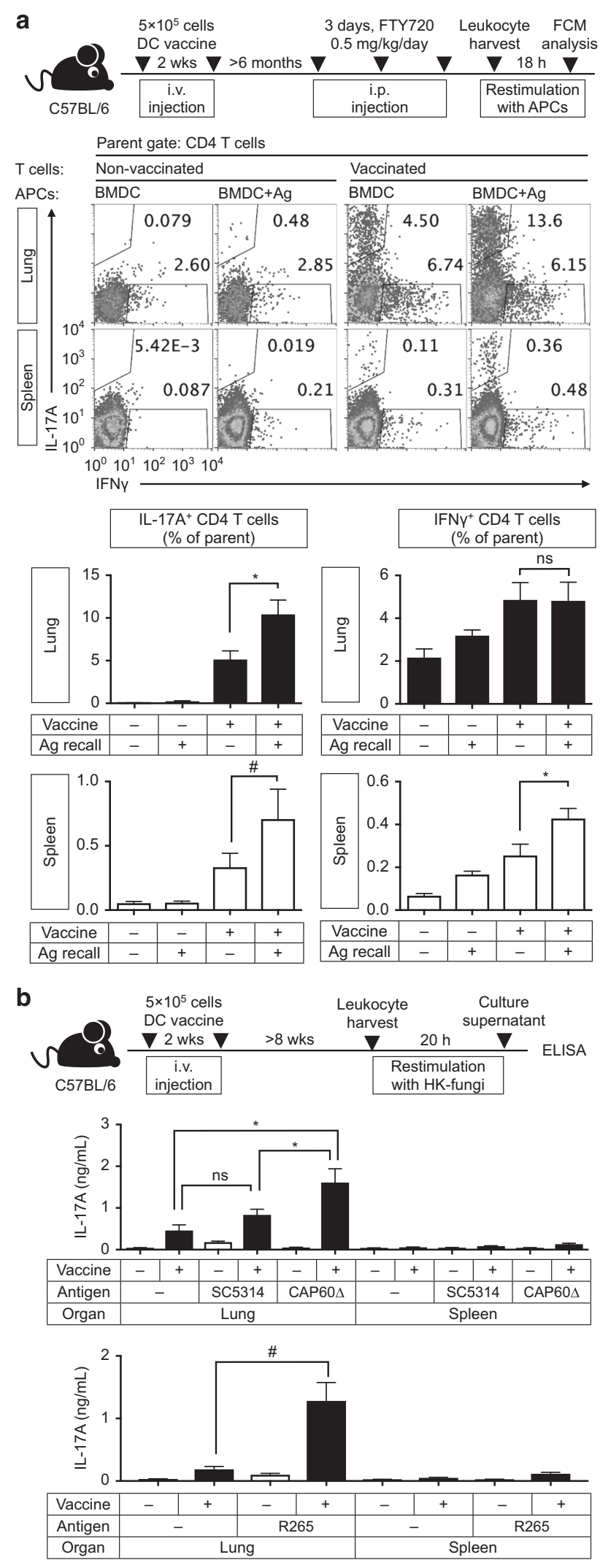

induction of neutrophils at day 7 post-infection is correlated with the effector function of lung TMR17.

Previous studies suggest that neutrophils are required for the induction of multinucleated giant cells (MGCs) that can suppress
Fig. 4 Vaccine-induced lung-resident $\mathrm{CD}^{+} \mathrm{T}$ cells carry memory of C. gattii-derived antigens. a Vaccine-induced lung-resident $\mathrm{CD} 4^{+}$ T cells increased production of IL-17A but not IFN $\gamma$ after antigen restimulation. Six months after the final vaccination, FTY720 was administrated to deplete circulating lymphocytes as described in Fig. 3. On the following day, the lungs and spleen were dissected to prepare leukocytes. Antigen (Ag)-pulsed BMDCs were used as antigen-presenting cells (APCs) to stimulate T cells. BMDCs $\left(5 \times 10^{5}\right.$ cells/well) were stimulated with antigen (heat-killed C. gattii CAP60 $\Delta$, $\mathrm{MOI}=1$ ) overnight. After the culture supernatant was removed, lung leukocytes or spleen leukocytes $\left(1 \times 10^{6}\right.$ cells/well) were added and co-cultivated for $18 \mathrm{~h}$. For the control, non-vaccinated/FTY720treated naive leukocytes and antigen-unloaded BMDCs were used. For flow cytometry analysis, gates were set for $\mathrm{CD} 4^{+}$Thy $1.2^{+}$cells. Representative flow cytometry profiles and bar graphs for statistical analysis are shown. Pooled data from two independent experiments were used to prepare bar graphs (means \pm SEMs, $n=8$ ). ${ }^{*} P<0.05$ in unpaired $t$-test; ${ }^{\#} P<0.05$ in Mann-Whitney $U$ test; ns no significant difference. b Vaccine-induced lung leukocytes produced higher amounts of IL-17A than spleen leukocytes in ex vivo re-stimulation assay. Eight weeks after the final vaccination, leukocytes $\left(1 \times 10^{6}\right.$ cells/well) were stimulated for $20 \mathrm{~h}$ with heat-killed (HK) fungal cells including C. albicans SC5314 $(\mathrm{MOI}=1)$, C. gattii CAP60 $2(\mathrm{MOI}=1)$, and C. gattii R265 $(\mathrm{MOI}=10)$. After cultivation, IL-17A in culture supernatant was measured using ELISA. For the control, leukocytes were harvested from the mice that received DC without antigen loading. Pooled data from more than two independent experiments were used to prepare bar graphs (means \pm SEMs, $n=7-10$ ). ${ }^{*} P<0.05$ in analysis of variance with Tukey's post hoc test; ${ }^{\#} P<0.05$ in unpaired $t$-test; ns no significant difference

the growth of Mycobacterium tuberculosis ${ }^{27}$. IL-17A-dependent granuloma formation is also known in murine tuberculosis ${ }^{28}$, cryptococcosis $^{29}$, and hypersensitivity pneumonitis models ${ }^{25}$. Our previous study showed that MGC formation was correlated with protective response against pulmonary infection with highly virulent $C$. gattii ${ }^{7}$. Therefore, we performed a histological evaluation of lung sections to assess MGC formation and localization of neutrophils at day 14 post-infection (Fig. 8). In these experiments, the WT and IL-17A KO mice were continuously treated by FTY720 to deplete circulating lymphocytes during the challenge, as described in Fig. $6 \mathrm{~d}$, e. In the unimmunized mice, many fungal cells were found in destroyed alveolar spaces, but the leukocyte accumulation was limited. The DC vaccine increased leukocytes in the lungs of the WT mice, and induced the formation of MGCs, which engulfed the fungal cells. However, accumulation of leukocytes and MGC formation were significantly lesser in the $\mathrm{IL}-17 \mathrm{~A}$ KO mice that received the DC vaccine. Larger alveolar spaces were also observed in the immunized IL-17A KO mice (Fig. 8a). In the lungs stained with hematoxylin-eosin (HE), infiltration of polymorphonuclear leukocytes (PMNs) and lymphocytes was found in the inflammatory region of the immunized mice, and these cells adjacently localized with MGCs (Fig. 8a). Immunohistochemical analysis also showed that considerable Ly6G ${ }^{+}$neutrophils adjacently localized with T cells in the lungs of the immunized mice in an IL-17A-dependent manner (Fig. 8b). These results suggest that lung TRM17 is involved in neutrophil recruitments and MGC formation at day 14 post-infection, which might suppress fungal growth in the lungs after infection.

\section{DISCUSSION}

In this study, we demonstrated that a systemic DC vaccine induced lung-resident memory Th17 cells (lung TRM17), which had a characteristic marker profile, antigen-specific memory, and a long life. Our results suggest that lung TRM17 is involved in the induction of neutrophils and MGCs in the lungs after pulmonary infection with the highly virulent $C$. gattii strain R265. This inflammatory response might be critical for long-term protective 
a Parent gate: CD69 ${ }^{+}$CD4 TRMs

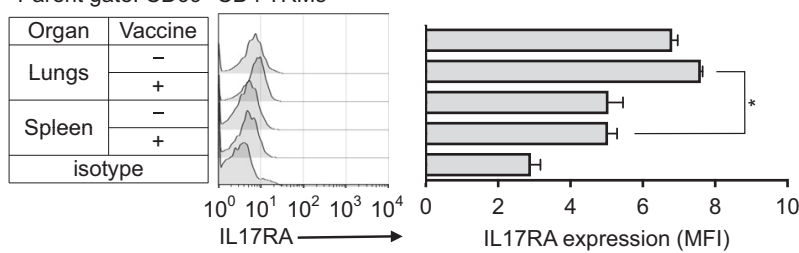

b
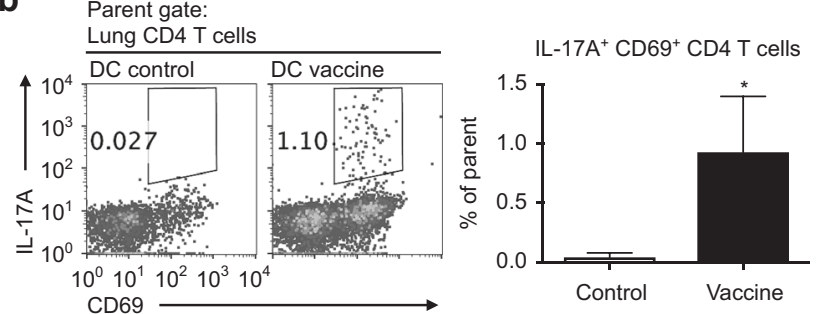

C

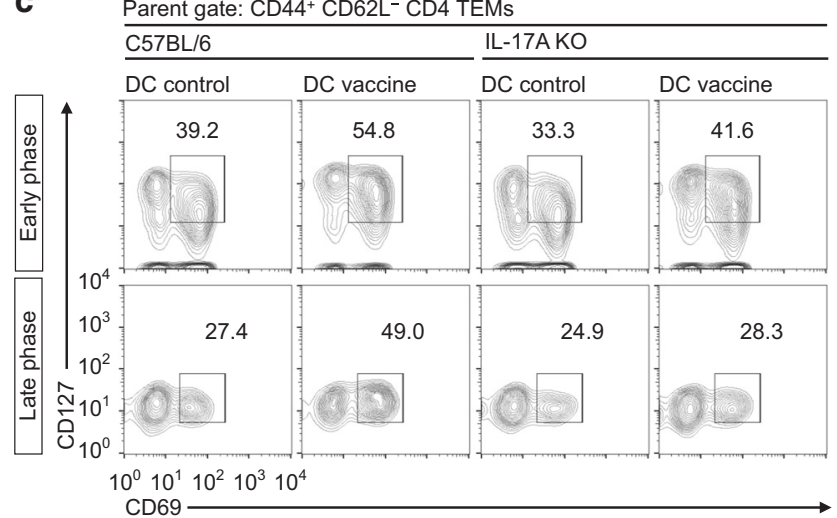

Parent gate: $\mathrm{CD}_{4} 4^{+} \mathrm{CD} 62 \mathrm{~L}-\mathrm{CD} 4 \mathrm{TEMs} \quad \square \mathrm{DC}$ control $\square \mathrm{DC}$ vaccine $\mathrm{CD} 127^{+} \mathrm{CD} 69^{+} \mathrm{CD} 4 \mathrm{TRMs}$

$\begin{array}{llll}\% \text { of parent Cells/lungs } & \text { CD69 MFI } & \text { CD127 MFI }\end{array}$
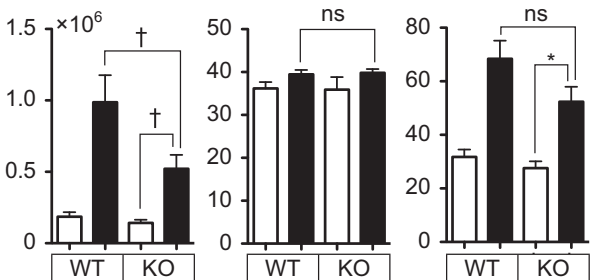

$\%$ of parent

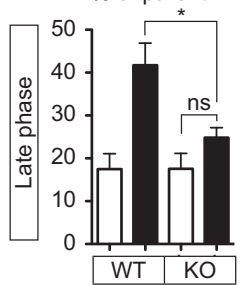

Cells/lungs
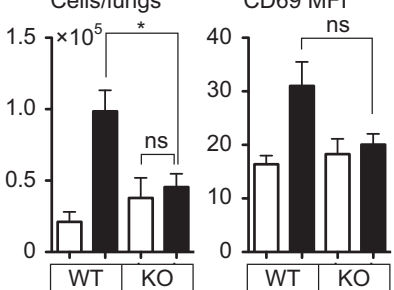

CD127 MFI

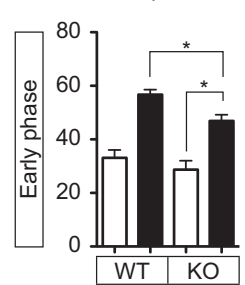

effect of the DC vaccine. Our findings provide new insights into an efficient approach for induction of lung-resident memory Th17 cells and a vaccination strategy against pulmonary infection, including the lethal C. gattii infection.

TRM-function of Th1, Th2, and CD8 ${ }^{+}$cytotoxic lymphocytes has been well-studied. It has been shown that local infection providing cognate antigens was essential to induce TRMs ${ }^{30}$. On the other hand, few studies investigated memory Th17 cells, and there is even a report indicating that Th17 cells are shorter-lived
Fig. 5 IL-17A was required for the development and maintenance of lung TRM17. a Lung TRM17 expressed the IL-17A receptor, IL-17RA. $\mathrm{CD}^{+}$TRMs in the lungs and spleen were analyzed by flow cytometry $>2$ weeks after the final vaccination. Representative flow cytometry profiles and bar graphs (means $\pm S D s, n=3$ ) from three independent experiments are shown. ${ }^{*} P<0.05$ in unpaired $t$-test. $\mathbf{b}$ Lung TRM17 produced IL-17A without antigen stimulation. Lung leukocytes were harvested $>6$ months after the final vaccination and were cultivated in medium for overnight without antigen to measure cytokine production. Representative flow cytometry profiles and bar graphs (means \pm SDs, $n=4$ ) from two independent experiments are shown. ${ }^{*} P<0.05$ in unpaired $t$-test. c The amount of lung TRM17 was decreased to non-vaccinated baseline within 15 weeks after the vaccination. Vaccination was performed as described above. Lung TRM17 was analyzed by flow cytometry after 2 weeks (early phase) and 15 weeks (late phase) of the final vaccination. Representative flow cytometry profiles are shown. Pooled data from two independent experiments were used to prepare bar graphs (means \pm SEMs, $n=6$ ). ${ }^{*} P<0.05$ in analysis of variance with Dunnett's post hoc test; ${ }^{\dagger} P<0.05$ in analysis of variance with Holm-Sidaks's post hoc test; ns no significant difference

than Th1 cells ${ }^{31}$. A recent report showed that intranasal vaccination induced lung-resident memory Th17 cells to promote local lgA responses ${ }^{32}$. An important distinction in our findings was that systemic vaccination also induced long-lived lung TRM17 with protective effects against pulmonary infection.

To the best of our knowledge, this is the first study showing that TRMs can be induced without local antigen administration or local infection. Our findings raise the question of why the systemic DC vaccine induced TRMs in the lungs rather than in BM or spleen. We showed that antigen-pulsed DCs preferentially accumulated in the lungs after intravenous transfer (Fig. 1). This migration ability might be crucial in inducing TRMs in the lungs. Shimizu et al. demonstrated that intravenous transfer of a-galactosylceramideloaded DCs induced long-lived invariant natural killer T cells in lungs, and transferred DCs also preferentially migrated to the lungs rather than to the spleen or liver ${ }^{33}$. There were reports showing that i.v.-injected BM-derived dendritic cells (BMDCs) preferentially migrated to the liver ${ }^{34}$ or spleen ${ }^{35,36}$. Such diverse tissue tropism might be attributable to the BMDC induction method. Adaptive-transferred DCs migrated to the liver or spleen rather than to the lungs when IL-4 was used with GM-CSF to cultivate BMDCs ${ }^{34,35}$. Specific culture conditions possibly allow the expression of specific chemokine receptors controlling DC trafficking. Administration of antigen-loaded DCs has been utilized not only in infection control ${ }^{11}$ but also in cancer therapy ${ }^{10}$. Tissueresident lymphocytes including TRMs and TILs are also involved in ameliorating other infections and cancer, respectively. Thus, the lung-targeting $D C$ vaccine might possess the potential to induce lung TRMs and lung TILs that can control respiratory infections and growing tumors. Future studies are needed to develop ways to regulate organ-specific migration of transferred DCs.

Our findings suggest that MHC-II on donor DCs is partially required for the generation of lung $\mathrm{CD}^{+}{ }^{+}$TRMs and that recruited endogenous DCs are also involved in the development of lung $\mathrm{CD}^{+}{ }^{+}$TRMs (Fig. 2c). In the current study, the heat-inactivated fungal cells were used to pulse DCs for preparing the DC vaccine. Because the fungal particles could not be removed from the DC suspensions by centrifugation and washing, the DC vaccine actually comprised BMDC engulfing the fungal cells and the extracellular non-engulfed fungal particles as previously described ${ }^{7}$. Thus, a considerable number of fungal particles were also injected via the tail vein into the host, while vaccination using heat-inactivated C. gattii strains without DCs had no protective effect $^{7}$. As shown in Fig. 1, the DC vaccine significantly recruited endogenous DCs; thus, it is likely that the endogenous DCs 


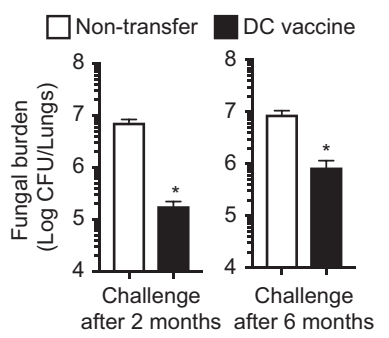

b $\quad$ Non-transfer

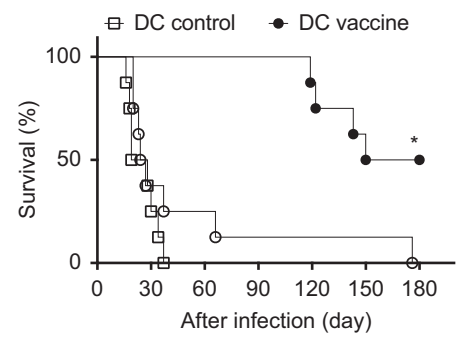

C
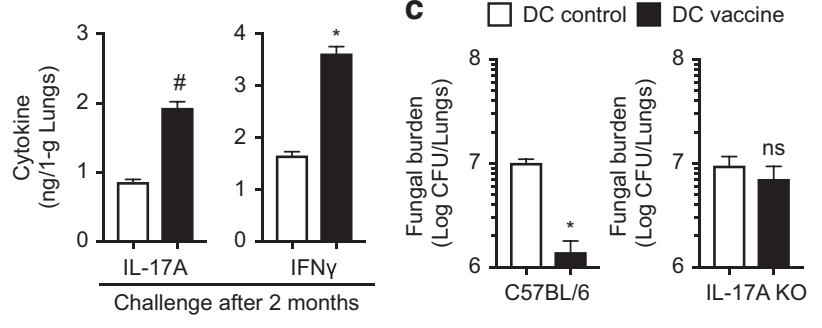

e
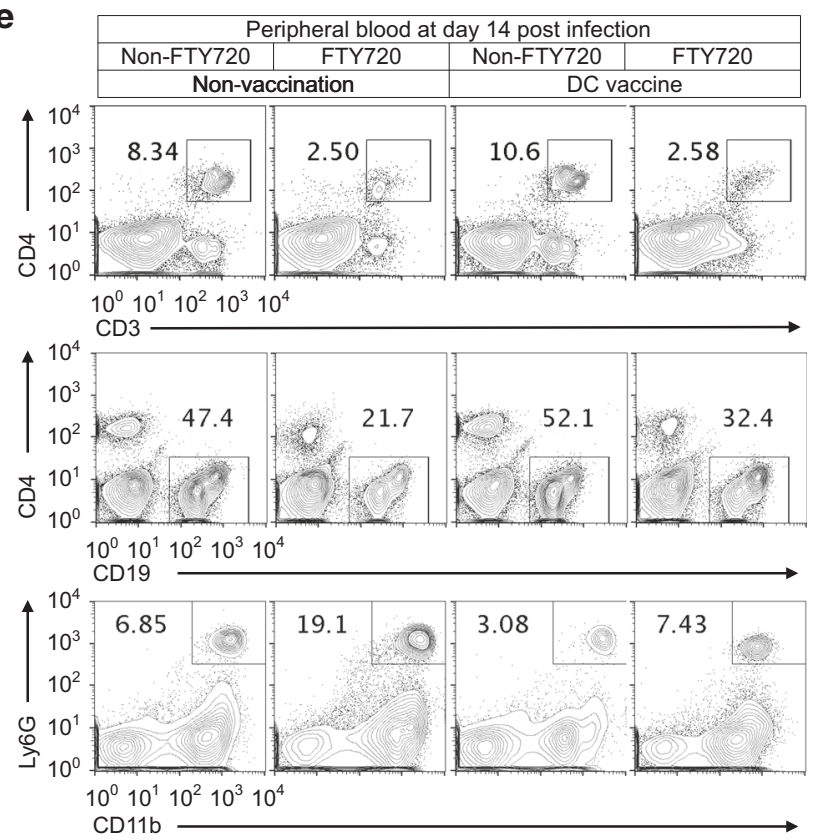

d

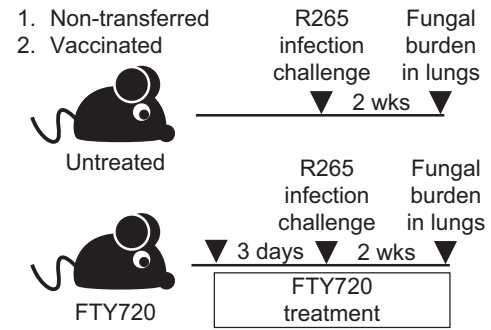

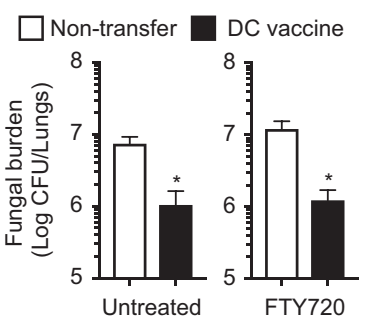

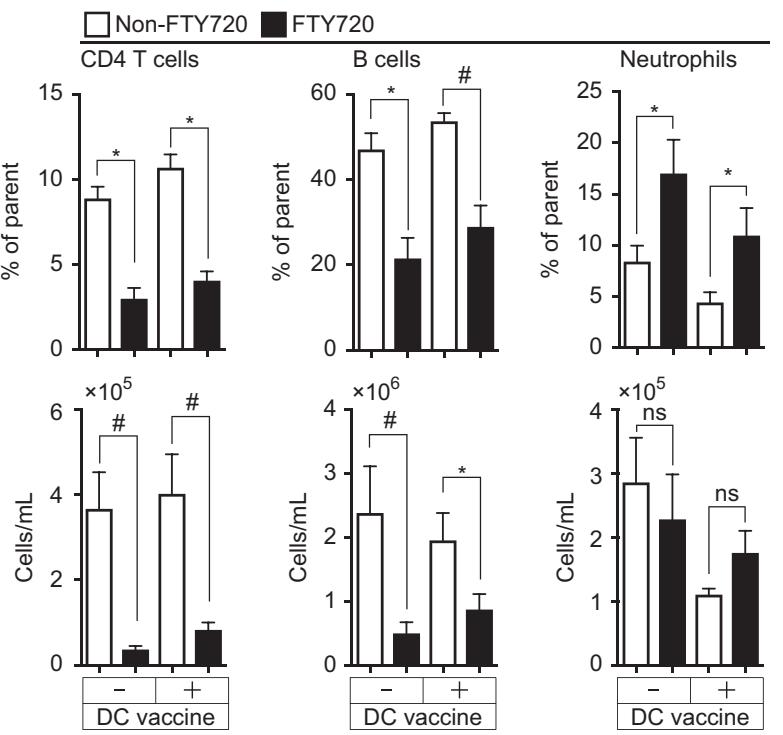

Fig. 6 Lung TRM17 was involved in the vaccine-mediated protective effect against infection with highly virulent $C$. gattii. a The DC vaccine suppressed fungal growth at day 15 post-infection, and the effect was sustainable for 6 months. Vaccination was performed as described above. Next, the highly virulent C. gattii strain R265 $\left(3 \times 10^{3}\right.$ cells/mouse) was intratracheally injected 2 or 6 months after the final vaccination. Lung homogenate was harvested at day 15 of post-infection. Pooled data from two independent experiments were used to prepare bar graphs (means \pm SEMs, $n=10$ ). ${ }^{*} P<0.05$ in unpaired $t$-test with Welch's correction; ${ }^{\#} P<0.05$ in unpaired $t$-test versus non-transfer control. b The DC vaccine improved the survival of $C$. gattii-infected mice. The infection challenge was performed after 2 months from final vaccination. Representative survival curve from two independent experiments are shown $(n=8)$. Median survival times were 25.5 days (nontransfer control), 23.5 days (DC control: transfer of DCs without antigen unloading), and 165 days (DC vaccine: transfer of CAP60 $\Delta$-pulsed DCs). ${ }^{*} P<0.05$ in log rank test versus non-transfer and DC controls. $c$ The DC vaccine suppressed fungal growth in an IL-17A-dependent manner. The infection challenge was performed 2 months after the final vaccination. Pooled data from two independent experiments were used to prepare bar graphs (means $\pm \mathrm{SEMs}, n=9-10$ ). ${ }^{*} P<0.05$ in unpaired $t$-test with Welch's correction versus DC control; ns no significant difference. d Circulating lymphocytes were dispensable for vaccine-mediated fungal growth suppression. The infection challenge was performed 6-10 weeks after the final vaccination. The FTY720-treated mice received $2.5 \mathrm{mg} / \mathrm{L} \mathrm{FTY720} \mathrm{in} \mathrm{drinking} \mathrm{water} \mathrm{ad} \mathrm{libitum} \mathrm{and} 1$ mg/ kg FTY720 via i.p. injection every 3-4 days for the duration of the experiment. Administration was started 3 days prior to the infection challenge. Pooled data from two independent experiments were used to prepare bar graphs (means \pm SEMs, $n=9-10$ ). ${ }^{*} P<0.05$ by unpaired $t$-test with Welch's correction versus non-transfer control; ns no significant difference. e Vascular CD4 ${ }^{+}$T cells and B cells, but not neutrophils, were depleted by FTY720 treatment at day 14 of post-infection. Peripheral blood leukocytes were analyzed by flow cytometry to validate the efficacy of FTY720 administration, as described in (d). Representative flow cytometry profiles and bar graphs for statistical analysis are shown. Pooled data from two independent experiments were used to prepare bar graphs (means \pm SEMs, $n=8$ ). ${ }^{*} P<0.05$ in unpaired $t$-test; ${ }^{\#} P<0.05$ in unpaired $t$-test with Welch's correction; ns no significant difference 

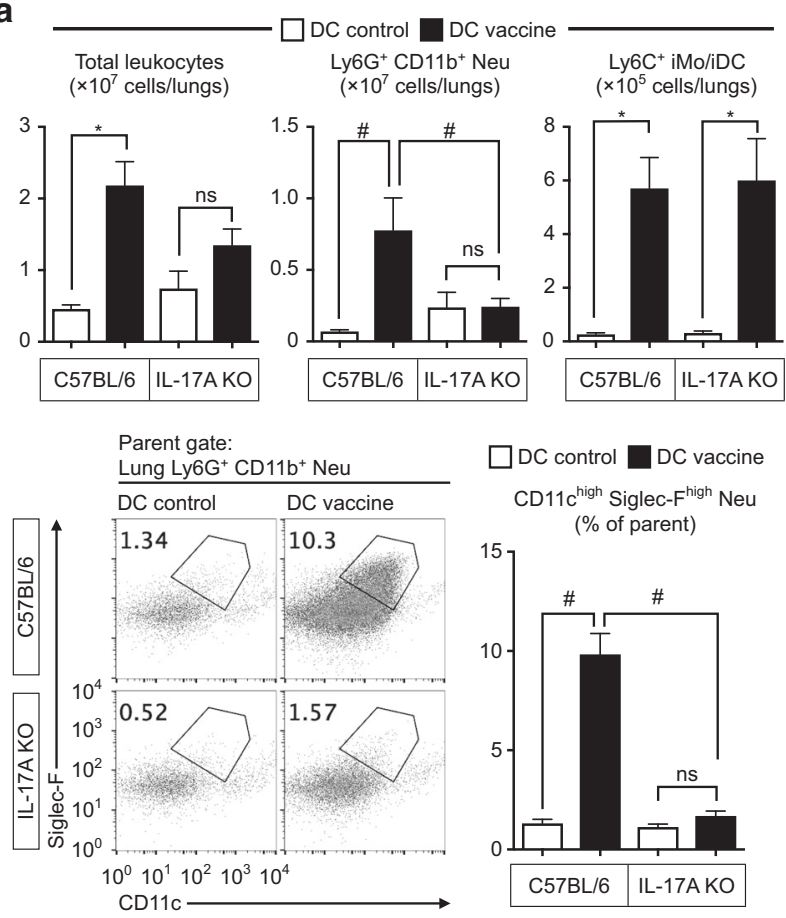

b
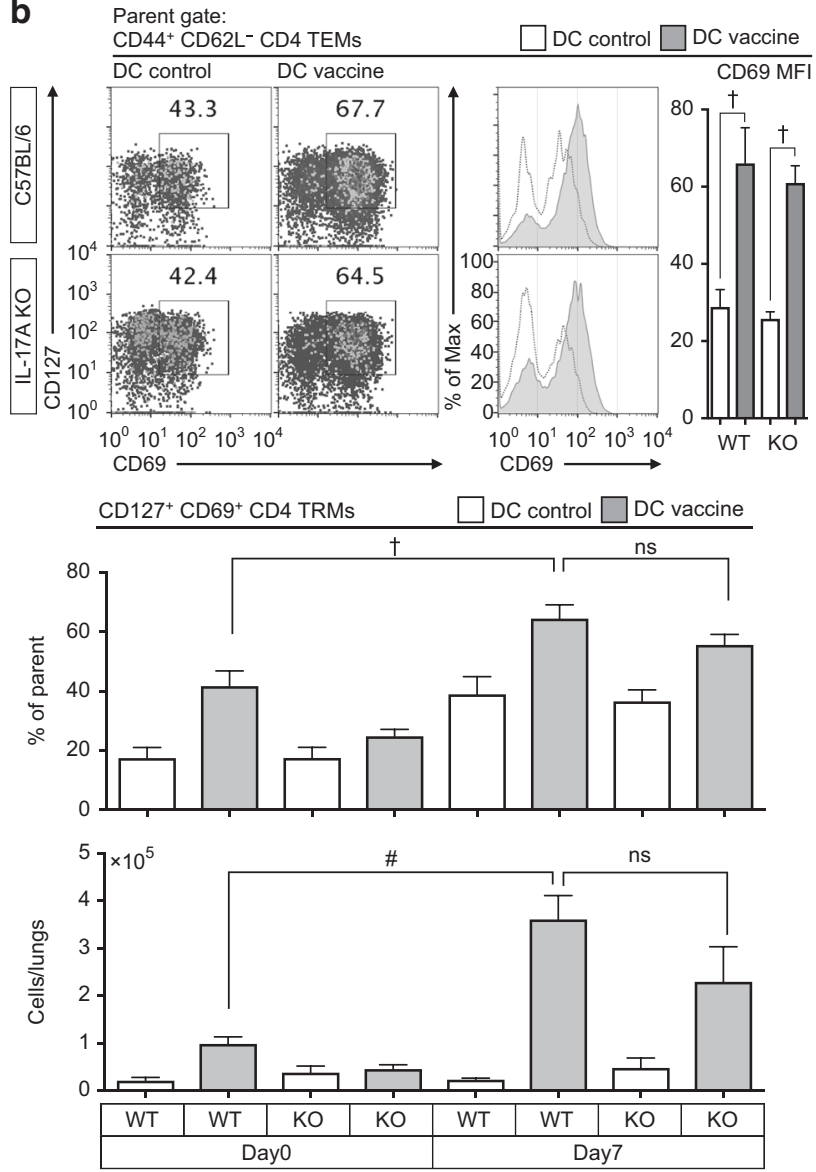

trapped the intravascular fungal particles for priming $\mathrm{CD}^{+}{ }^{+} \mathrm{T}$ cells during the vaccination.

We showed that DC vaccine-mediated lung TRM17 were longlived in the lungs, until at least 6 months after the immunization
Fig. 7 Lung TRM17 was involved in activation of neutrophils at day 7 post-infection. The infection challenge was performed $>4$ months after the final vaccination. To evaluate lung TRM17-mediated inflammatory response, all mice continuously received FTY720 for the duration of the experiment as described in Fig. $6 \mathrm{~d}$, e. Lung leukocytes were analyzed using flow cytometry at day 7 postinfection. a Gates were set for neutrophils (Neu: Ly6G ${ }^{+}$CD11b cells) and inflammatory monocytes/dendritic cells (iMo/iDC: Ly6G Siglec- $\mathrm{F}^{-}$MHC- $\mathrm{II}^{+} \mathrm{CD}_{11 \mathrm{c}^{+}} \mathrm{Ly}_{6 \mathrm{C}^{+}} \mathrm{CD}_{11 \mathrm{~b}^{+}}$cells) as described previously ${ }^{19,20}$. Because the population of Siglec- $F^{\text {high }}$ Ly6G low SSC high eosinophils and alveolar macrophages were outside of the neutrophil-gate, neutrophils expressing Siglec-F and CD11c were clearly distinguishable from other cells. b Gates were set for $\mathrm{CD}^{+}$ TEMs as described above, and the CD69 expression level was measured to evaluate the activation status of $\mathrm{CD} 4^{+}$TRMs. Pooled data from two independent experiments were used to prepare bar graphs (means \pm SEMs, $n=6$ ). ${ }^{\top} P<0.05$ in unpaired $t$-test; ${ }^{*} P<0.05$ in unpaired $t$-test with Welch's correction; ${ }^{\#} P<0.05$ in analysis of variance with Dunnett's post hoc test; ns no significant difference

(Fig. 4 and Supplementary Figure S2). There have been reports stating that long-lived memory $\mathrm{T}$ cells are $\mathrm{BCL}-2^{+}$and antiapoptotic ${ }^{37}$ Ki-67 negative and resting ${ }^{38}$, autophagyactivated $^{39,40}, \mathrm{Wnt} / \beta$-catenin pathway-activated ${ }^{41}$, or dependent on Notch signal-regulated glucose uptake and metabolism ${ }^{42}$ and lipid-uptake and metabolism ${ }^{43}$. Lung TRM17 highly expressed Ki67, TCF-7, stem cell antigen-1 (Sca-1), and the stem cell marker CD43 (Supplementary Figure S3C). TCF-7 is a Wnt signal-mediated transcriptional factor associated with self-renewal of stem cells ${ }^{44}$ and stem cell-like memory T-cells ${ }^{45}$. Although it was unknown whether the DC vaccine-mediated lung TRM17 was capable of self-renewal, stem cell-like marker profile might be responsible for the long-lived nature of lung TRM17. Future studies are also needed to elucidate the precise molecular mechanism for the long-lived nature of lung TRM17.

lijima and Iwasaki showed that vaginal-resident $\mathrm{CD}^{+}{ }^{+} \mathrm{TRMs}$ produced a basal level of IFNy to maintain themselves in the memory lymphocytes cluster (MLC), which establishes a feedback loop with $\mathrm{CD}_{11} \mathrm{~b}^{+}$macrophages secreting CCL5. Thus, IFNy and CCL5 were required for the maintenance of $C D 4^{+}$TRMs within the $\mathrm{MLC}^{46}$. We showed that IL-17A production was required for the generation and maintenance for lung TRM17 and that vaccineinduced lung TRM17 could produce IL-17A without antigen restimulation and expressed IL-17A receptor IL-17RA (Figs. 4 and 5). The amount of lung TRM17 in the IL-17A KO mice decreased to non-vaccinated baseline, and CD127 expression on lung CD4 ${ }^{+}$ TEMs was significantly attenuated in these within 15 weeks of the final vaccination (Fig. $5 \mathrm{C}$ ). Thus, IL-17A production appears to be required for CD127 expression on lung TRM17. Previous studies have shown that CD127 is a receptor for IL-7 and is responsible for the survival and generation of memory $\mathrm{CD}^{+} \mathrm{T}^{+}$cells ${ }^{47}$. Future studies are needed to elucidate how paracrine or autocrine IL-17A signals are involved in the generation and maintenance of lung TRM17.

$\mathrm{CD}^{+}$TRMs are basically helper cells and probably do not directly kill pathogens or tumor cells. Although the role of IFNy producing $\mathrm{CD}^{+}{ }^{+}$TRMs has been often analyzed ${ }^{48}$, the substantial role of IL-17A-producing CD4 ${ }^{+}$TRMs has yet to be elucidated. Th1type skin-resident memory $T$ cells play a role in the induction of inflammatory monocytes after re-infection in a parasite infection model $^{48}$. On the other hand, intranasal immunization-mediated memory Th17 cells in the lungs promoted local IgA response, but did not show protective effects against any respiratory infections including viral infections ${ }^{32}$. The DC vaccine-mediated lung TRM17 promptly responded to the antigen re-stimulation and produced IL-17A but not IFNy (Fig. 4). Furthermore, lung TRM17 appeared to induce neutrophils and MGCs after the infection challenge, which may have contributed to the vaccine-mediated protective effect 

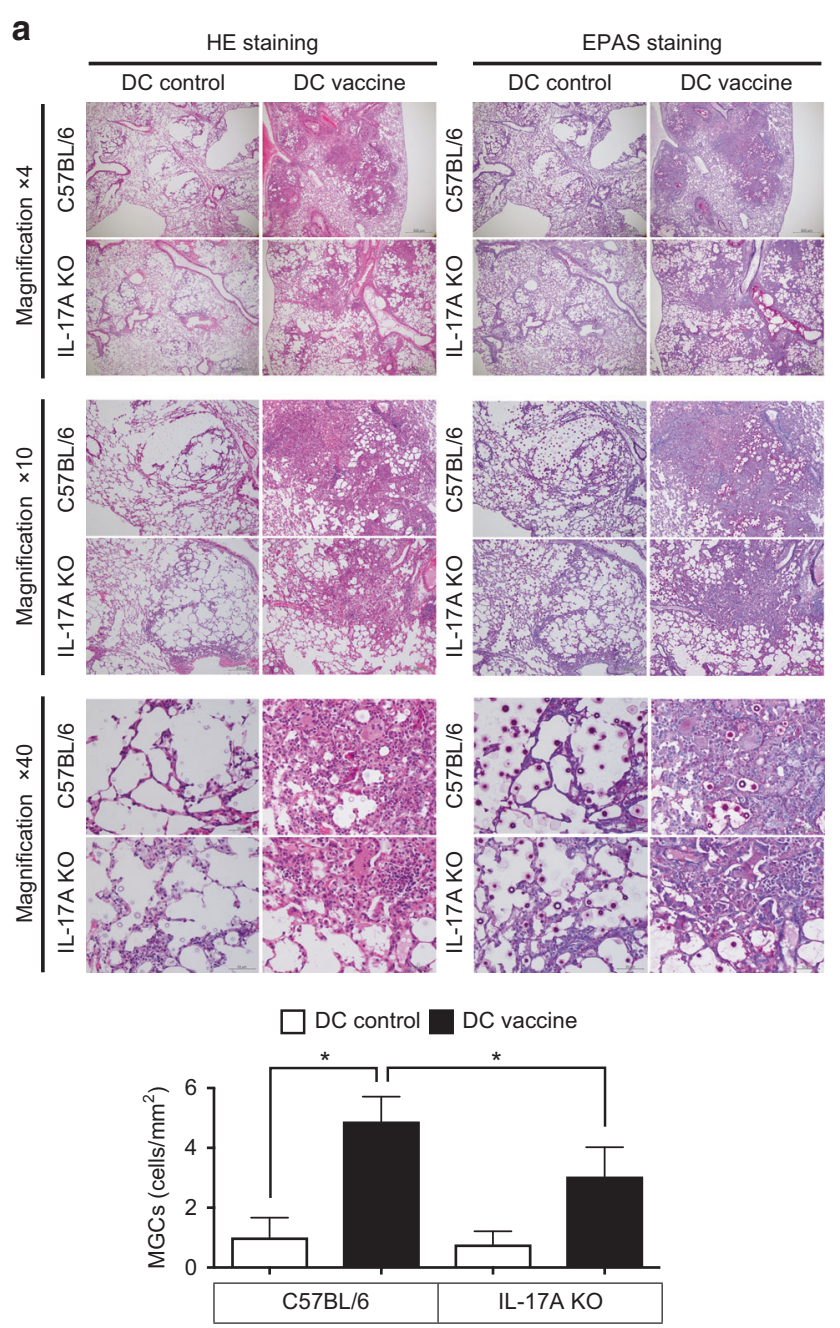

b

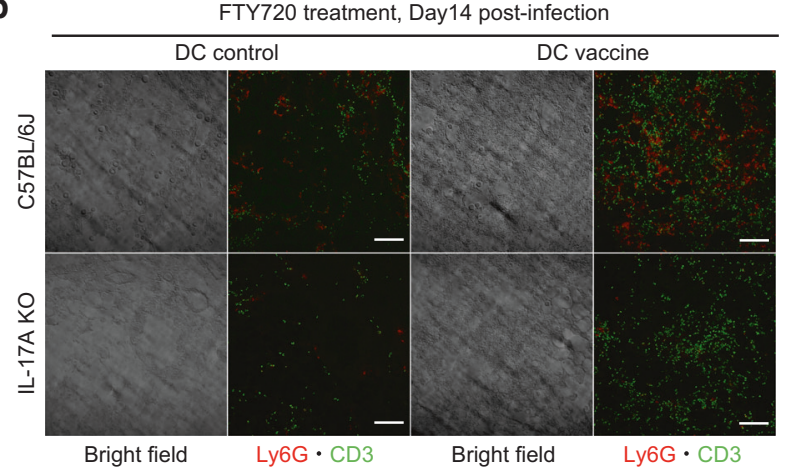

Fig. 8 Lung TRM17 was involved in the neutrophil recruitment and MGC formation at day 14 post-infection. a DC vaccine induced MGC formation in an IL-17A-dependent manner. Paraffin sections $(4 \mu \mathrm{m})$ were stained with HE or EPAS. The number of MGCs per unit area $\left(\mathrm{mm}^{2}\right)$ was evaluated, and relevant bar graphs (means \pm SDs, $n=3$ ) are shown. ${ }^{*} P<0.05$ in analysis of variance with Dunnett's post hoc test. b Neutrophils adjacently localized with T cells in an IL-17Adependent manner. Vaccination, FTY720-administration, and the infection challenge were performed as described in Fig. 7. The lungs were fixed in fixation buffer (2\% PFA in $50 \mathrm{mM}$ phosphate buffer) and then embedded in Tissue-Tek OCT compound. After freezing the organ blocks, $8-\mu \mathrm{m}$ thin sections were prepared and stained with the antibodies depicted in the figure. The sections were observed under confocal laser microscopy. Bar $=100 \mu \mathrm{m}$ against highly virulent C. gattii (Figs. 6-8). A Th17-inducing intranasal vaccine also promoted granuloma formation in an IL-17A- and a CXCL13-dependent manner and exhibited a protective effect against $M$. tuberculosis ${ }^{28}$. To the best of our knowledge, this is the first study elucidating a substantial role for Th17-type lung-resident memory $T$ cells.

In summary, we demonstrated that a systemic DC vaccine induced long-lived lung-resident memory Th17 cells. A Th17inducing vaccine is especially awaited in respiratory infections, including tuberculosis, pneumococcal infection, pertussis, and flu ${ }^{6}$. To further elucidate the precise role of lung TRM17, parabiosis and adoptive transfer experiments of $\mathrm{CD}^{+}{ }^{+}$TRMs are required in future research as it is currently difficult to specifically deplete TRMs. We believe that the findings of this study will provide important clues for the development of effective vaccines against intractable pulmonary infections.

\section{MATERIALS AND METHODS \\ Mice}

C57BL/6J (wild-type, WT) and B6.SJL-Ptprca Pepcb/BoyJ (CD45.1) were purchased from Japan SLC, Inc. (Shizuoka, Japan), and Sankyo Labo Service Corporation (Tokyo, Japan), respectively. The IL-17A KO mice were generated as described previously ${ }^{49}$. All mice were of the C57BL/6J background. The mice used in this study were sex-matched and age-matched and maintained under specific-pathogen-free conditions at the National Institute of Infectious Diseases of Japan.

\section{DC vaccine}

The DC vaccine was prepared and injected as previously described $^{7}$. In brief, BM cells were harvested from the femurs and tibiae of female mice. Red blood cells were lysed with lysis buffer $(15 \mathrm{mM}$ ammonium chloride, $1 \mathrm{mM}$ sodium bicarbonate, and $0.1 \mathrm{mM}$ ethylenediaminetetraacetic acid [EDTA]/bisodium, $\mathrm{pH}$ 7.4). The BM cells were cultured in RPMI 1640 complete medium that was supplemented with $10 \%(\mathrm{v} / \mathrm{v})$ fetal bovine serum (FBS), $1 \%(v / v)$ streptomycin-penicillin solution (Sigma-Aldrich, St. Louis, $\mathrm{MO}), \quad 44 \mu \mathrm{M}$ 2-mercaptoethanol, and $10 \mathrm{ng} / \mathrm{mL}$ of mouse granulocyte-macrophage colony-stimulating factor (mGM-CSF, PeproTech, Rocky Hill, NJ) at $37^{\circ} \mathrm{C}$ under $5 \% \mathrm{CO}_{2}$. On days 3 and 5 , medium was changed to maintain the BM cells. On day 6 , non-adherent cells were collected and used as BMDCs. BMDCs were incubated in RPMI 1640 complete medium with heat-killed C. gattii CAP60 $\triangle(\mathrm{MOI}=5)$ for $24 \mathrm{~h}$ in petri dishes. After incubation, non-adherent cells were collected and washed twice with phosphate buffered saline (PBS). BMDCs pulsed with heat-killed C. gattii CAP60 $\triangle\left(5 \times 10^{5}\right.$ cells/mouse) were injected twice every 2 weeks via the tail vein. For the blocking of MHC-II, antigenpulsed DCs were treated with $10 \mu \mathrm{g} / \mathrm{mL}$ anti-I-A/I-E mAb (M15/ 114.15.2, BioLegend, San Diego, CA) or rat IgG2b isotype control antibody (RTK4530, BioLegend, San Diego, CA) and washed twice with PBS.

\section{FTY720 treatment and intravascular staining}

FTY720 (Cayman Chemical, Ann Arbor, MI) was dissolved in dimethyl sulfoxide (DMSO) to prepare a $50 \mathrm{mg} / \mathrm{mL}$ stock solution. The stock solution was diluted with saline and distilled water for intraperitoneal administration $(0.5-1.0 \mathrm{mg} / \mathrm{kg}$ ) and administration in drinking water $(2.5 \mathrm{mg} / \mathrm{L})$, respectively. To stain vascular leukocytes (IV staining), $2.5 \mu \mathrm{g}$ of FITC-labeled anti-CD45 mAb (BioLegend, San Diego, CA) was injected into the orbital vein under isoflurane anesthesia $5 \mathrm{~min}$ prior to euthanasia.

Leukocyte preparation

Leukocytes in the BM, spleen, lungs, and MedLN were prepared as previously described ${ }^{7}$. In brief, the BM, spleen, and MedLN were mashed on a $70-\mu \mathrm{m}$ cell strainer, and red blood cells were then 
lysed with the lysis buffer, as described above. The lungs were minced and digested with collagenase-D, hyaluronidase and DNase I (Sigma-Aldrich, St. Louis, MO). Lung leukocytes were enriched with Percoll gradient (30\%, 44\%, and 70\%) centrifugation.

\section{Flow cytometry}

Flow cytometry was performed as previously described ${ }^{7}$. Antibodies used in this study are listed in Supplementary Table S1. Staining buffer was PBS containing $2 \mathrm{mM}$ EDTA, 0.5\% (w/v) bovine serum albumin, and $0.1 \%(\mathrm{w} / \mathrm{v})$ sodium azide. Fixation and permeabilization buffers were purchased from BioLegend (San Diego, CA). FOXP3/transcription factor staining buffer set (eBioscience, San Diego, CA) was used to stain transcription factors. Gating strategies used for lung leukocytes ${ }^{19,20}$ and memory $\mathrm{T}$ cells ${ }^{13}$ were as previously described. Data were acquired with a BD FACSCalibur or BD FACSCantoll flow cytometer (BD Bioscience, San Jose, CA) and analyzed using FlowJo software (TreeStar Inc., Ashland, OR).

\section{T-cell re-stimulation assay}

For polyclonal re-stimulation, the lung, spleen, and BM leukocytes were incubated in RPMI 1640 medium with $50 \mathrm{ng} / \mathrm{mL}$ of PMA and $1 \mu \mathrm{M}$ ionomycin for $4 \mathrm{~h}$. Alternatively, for the antigen-specific restimulation, BMDCs $\left(5 \times 10^{5}\right.$ cells/well $)$ were pulsed with heatkilled C. gattii CAP60 $\triangle\left(5 \times 10^{5}\right.$ cells/well) overnight. After the culture supernatant was removed, antigen-pulsed BMDCs were co-cultivated with lung leukocytes or spleen leukocytes $\left(1 \times 10^{6}\right.$ cells/well) for $18 \mathrm{~h}$ to stimulate T-cell response. In each experiment, cells were treated with $5 \mu \mathrm{g} / \mathrm{mL}$ brefeldin $A$ and $2 \mu \mathrm{M}$ monensin for the last $2 \mathrm{~h}$ of culture to stop cytokine release. Intracellular cytokines in $\mathrm{T}$ cells were evaluated using flow cytometry. In another experiment, lung and spleen leukocytes $\left(1 \times 10^{6}\right.$ cells/well) were cultivated for $20 \mathrm{~h}$ with heat-killed $C$. albicans SC5314 $(\mathrm{MOI}=1)$, C. gattii CAP60 $\Delta(\mathrm{MOI}=1)$, or C. gattii R265 $(\mathrm{MOI}=10)$. After cultivation, IL-17A in culture supernatant was measured using enzyme-linked immunosorbent assay (ELISA).

\section{Cytokine measurement}

Supernatants of cell culture or lung homogenates were collected and cytokine levels were determined using ELISA. MaxiSorp plates (Thermo Fisher Scientific, Roskilde, Denmark) and a DuoSet ELISA kit (R\&D Systems, Minneapolis, MN) or BD OptEIA ELISA sets (BD Bioscience, San Jose, $C A$ ) were used as per the manufacturers' instructions.

\section{Infection study}

Intratracheal infection was performed as previously described ${ }^{7}$. In brief, C. gattii R265 was cultured in YPD at $30^{\circ} \mathrm{C}$ with shaking overnight. Then the cells were washed and resuspended in PBS. The mice were anesthetized with isoflurane, after which a fungal suspension $\left(3 \times 10^{3} \mathrm{CFU}\right)$ was intratracheally injected using an indwelling needle. They were euthanized with carbon dioxide inhalation to harvest their lungs, and the lungs were homogenized using a stainless-steel mesh in PBS. To determine the fungal burden in lungs, lung homogenates were diluted and spread onto YPD plates. These plates were incubated at $30^{\circ} \mathrm{C}$ for $24 \mathrm{~h}$, after which colonies were counted.

\section{Histological analysis}

The lungs were fixed in $10 \%$ formalin, dehydrated, and embedded in paraffin. Paraffin blocks were cut into 4- $\mu \mathrm{m}$ sections and stained with HE or Verhoeff's elastin staining and periodic acid-Schiff's method (EPAS). MGCs were examined to assess macrophage recruitment into the lungs after infection. Numbers of MGCs per unit area (square millimeters) were determined as previously described $^{7}$. Frozen lung sections were prepared and stained as previously described ${ }^{50}$. Antibodies used in this experiment are also listed in Supplementary Table S1. Infected lungs were fixed in fixation buffer, 2\% (w/v)-paraformaldehyde (PFA) in $50 \mathrm{mM}$ phosphate buffer, for $6 \mathrm{~h}$ at $4{ }^{\circ} \mathrm{C}$ prior to be embedded in Tissue-Tek OCT compound (Sakura Finetek Inc., Torrance, CA). Fluorescent-labeled lung sections were observed under a confocal laser-scanning microscope LSM 700 (Carl Zeiss, Oberkochen, Germany), and the images were analyzed by ZEN software (Carl Zeiss, Oberkochen, Germany).

Statistical analysis

GraphPad Prism 7 (GraphPad Software, Inc., La Jolla, CA) was used for all statistical analyses. $P$-values of less than 0.05 were considered statistically significant.

\section{ACKNOWLEDGEMENTS}

This work was supported by KAKENHI $(15 \mathrm{~K} 21644,16 \mathrm{H} 05349$, and 17K18385) from the Ministry of Education, Culture, Sports, Science and Technology of Japan, by grants from the Japan Agency for Medical Research and Development, AMED, by the Takeda Science Foundation, and by the LEGEND Research Grant Program 2015 from Tomy Digital Biology Co. Ltd. We also thank Drs. Yu Adachi, Taishi Onodera, Yoshimasa Takahashi, and Koji Hayashizaki who provided the technical advice for immunohistochemical analysis.

\section{AUTHOR CONTRIBUTIONS}

K.U. designed the experiments. K.U., M.U., S.S., M.S., S.T., M.A., Y.O. and N.Y. conducted the experiments. K.U. and Y.K. analyzed the data and wrote the article. K.U., K. Shimizu, Y.I., K. Shibuya, Y.M. and Y.K. interpreted the data, and all authors reviewed and approved the manuscript.

\section{ADDITIONAL INFORMATION}

The online version of this article (https://doi.org/10.1038/s41385-018-0094-4) contains supplementary material, which is available to authorized users.

Competing interests: The authors declare no competing interests.

Ethical statement:All animal experiment protocols were approved by the Ethical Committee of the National Institute of Infectious Diseases, Japan (approval numbers $115015,116019,116124,215031,215036,215047,114029,117032$, and 114029) and were performed in accordance with the approved guidelines and regulations.

\section{REFERENCES}

1. Mueller, S. N. \& Mackay, L. K. Tissue-resident memory T cells: local specialists in immune defence. Nat. Rev. Immunol. 16, 79-89 (2016).

2. Park, C. O. \& Kupper, T. S. The emerging role of resident memory T cells in protective immunity and inflammatory disease. Nat. Med. 21, 688-697 (2015).

3. Ganesan, A.-P. et al. Tissue-resident memory features are linked to the magnitude of cytotoxic T cell responses in human lung cancer. Nat. Immunol. 18, 940-950 (2017).

4. Hayashizaki, K. et al. Myosin light chains 9 and 12 are functional ligands for CD69 that regulate airway inflammation. Sci. Immunol. 1, eaaf9154 (2016).

5. Mackay, L. K. et al. Cutting edge: CD69 interference with sphingosine-1phosphate receptor function regulates peripheral T cell retention. J. Immunol. 194, 2059-2063 (2015).

6. Lin, Y., Slight, S. R. \& Khader, S. A. Th17 cytokines and vaccine-induced immunity Semin. Immunopathol. 32, 79-90 (2010).

7. Ueno, K. et al. Dendritic cell-based immunization ameliorates pulmonary infection with highly virulent Cryptococcus gattii. Infect. Immun. 83, 1577-1586 (2015)

8. Lizarazo, J. et al. Retrospective study of the epidemiology and clinical manifestations of Cryptococcus gattii infections in Colombia from 1997-2011. PLoS Negl. Trop. Dis. 8, e3272 (2014).

9. CDC Emergence of Cryptococcus gattii, Pacific Northwest, 2004-2010. Morb. Mortal. Wkly. Rep. 59, 865-868 (2010).

10. Palucka, K. \& Banchereau, J. Dendritic-cell-based therapeutic cancer vaccines. Immunity 39, 38-48 (2013)

11. García, F. et al. A therapeutic dendritic cell-based vaccine for HIV-1 infection. J. Infect. Dis. 203, 473-478 (2011).

12. Roy, R. M. \& Klein, B. S. Dendritic cells in antifungal immunity and vaccine design. Cell Host Microbe 11, 436-446 (2012). 
13. Turner, D. L. \& Farber, D. L. Mucosal resident memory CD4 T cells in protection and immunopathology. Front. Immunol. 5, 331 (2014).

14. McKinstry, K. K. et al. Effector CD4 T-cell transition to memory requires late cognate interactions that induce autocrine IL-2. Nat. Commun. 5, 5377 (2014).

15. Takenaka, M. et al. Antibodies to MHC class II molecules induce autoimmunity: critical role for macrophages in the immunopathogenesis of obliterative airway disease. PLoS ONE 7, e42370 (2012).

16. Anderson, K. G. et al. Intravascular staining for discrimination of vascular and tissue leukocytes. Nat. Protoc. 9, 209-222 (2014).

17. Turner, D. L. et al. Lung niches for the generation and maintenance of tissueresident memory T cells. Mucosal Immunol. 7, 501-510 (2014).

18. Laidlaw, B. J. et al. $\mathrm{CD} 4(+) \mathrm{T}$ cell help guides formation of $\mathrm{CD} 103$ (+) lung-resident memory CD8(+) T cells during influenza viral infection. Immunity 41, 633-645 (2014).

19. Sionov, E. et al. Type I IFN induction via poly-ICLC protects mice against Cryptococcosis. PLoS Pathog. 11, e1005040 (2015).

20. Iwata, A. et al. Th2-type inflammation instructs inflammatory dendritic cells to induce airway hyperreactivity. Int. Immunol. 26, 103-114 (2013).

21. Lewis, S. M. et al. Expression of CD11C and EMR2 on neutrophils: potential diagnostic biomarkers for sepsis and systemic inflammation. Clin. Exp. Immunol. 182, 184-194 (2015).

22. Villa-Ambriz, J., Rodríguez-Orozco, A. R., Béjar-Lozano, C. \& Cortés-Rojo, C. The increased expression of $\mathrm{CD} 11 \mathrm{C}$ and $\mathrm{CD} 103$ molecules in the neutrophils of the peripheral blood treated with a formula of bacterial ribosomes and proteoglycans of Klebsiella pneumoniae. Arch. Bronconeumol. 48, 316-319 (2012).

23. Percopo, C. M. et al. SiglecF ${ }^{+} \mathrm{Gr}^{\mathrm{hi}}$ eosinophils are a distinct subpopulation within the lungs of allergen-challenged mice. J. Leukoc. Biol. 101, 321-328 (2017).

24. Kiwamoto, T., Kawasaki, N., Paulson, J. C. \& Bochner, B. S. Siglec-8 as a drugable target to treat eosinophil and mast cell-associated conditions. Pharmacol. Ther. 135, 327-336 (2012).

25. Ishizuka, M. et al. Interleukin-17A and neutrophils in a murine model of birdrelated hypersensitivity pneumonitis. PLoS ONE 10, e0137978 (2015)

26. Zhang, M. et al. Defining the in vivo function of Siglec-F, a CD33-related Siglec expressed on mouse eosinophils. Blood 109, 4280-4287 (2007).

27. Yoshioka, Y. et al. Neutrophils and the $\$ 100 \mathrm{~A} 9$ protein critically regulate granuloma formation. Blood Adv. 1, 184-192 (2016).

28. Gopal, R. et al. Interleukin-17-dependent CXCL-13 mediates mucosal vaccineinduced immunity against tuberculosis. Mucosal Immunol. 6, 972-984 (2013).

29. Murdock, B. J., Huffnagle, G. B., Olszewski, M. A. \& Osterholzer, J. J. Interleukin-17A enhances host defense against cryptococcal lung infection through effects mediated by leukocyte recruitment, activation, and gamma interferon production. Infect. Immun. 82, 937-948 (2014).

30. Khan, T. N., Mooster, J. L., Kilgore, A. M., Osborn, J. F. \& Nolz, J. C. Local antigen in nonlymphoid tissue promotes resident memory CD8 $+\mathrm{T}$ cell formation during viral infection. J. Exp. Med. 213, 951-966 (2016).

31. Pepper, M. et al. Different routes of bacterial infection induce long-lived TH1 memory cells and short-lived TH17 cells. Nat. Immunol. 11, 83-89 (2009).
32. Christensen, D., Mortensen, R., Rosenkrands, I., Dietrich, J. \& Andersen, P. Vaccineinduced Th17 cells are established as resident memory cells in the lung and promote local IgA responses. Mucosal Immunol. 10, 260-270 (2017).

33. Shimizu, K. et al. KLRG + invariant natural killer T cells are long-lived effectors. Proc. Natl. Acad. Sci. USA 111, 12474-12479 (2014).

34. Lappin, M. B. et al. Analysis of mouse dendritic cell migration in vivo upon subcutaneous and intravenous injection. Immunology 98, 181-188 (1999).

35. Mullins, D. W. et al. Route of immunization with peptide-pulsed dendritic cells controls the distribution of memory and effector T cells in lymphoid tissues and determines the pattern of regional tumor control. J. Exp. Med. 198, 1023-1034 (2003).

36. Sheasley-O'Neill, S. L., Brinkman, C. C., Ferguson, A. R., Dispenza, M. C. \& Engelhard, V. H. Dendritic cell immunization route determines integrin expression and lymphoid and nonlymphoid tissue distribution of CD8 T cells. J. Immunol. 178, 1512-1522 (2007).

37. Kryczek, I. et al. Human TH17 cells are long-lived effector memory cells. Sci. Transl. Med. 3, 104ra100 (2011).

38. Sercan Alp, Ö. et al. Memory CD8(+) T cells colocalize with IL-7(+) stromal cells in bone marrow and rest in terms of proliferation and transcription. Eur. J. Immunol. 45, 975-987 (2015)

39. $\mathrm{Xu}, \mathrm{X}$. et al. Autophagy is essential for effector CD8(+) T cell survival and memory formation. Nat. Immunol. 15, 1152-1161 (2014).

40. Araki, K. et al. mTOR regulates memory CD8 T-cell differentiation. Nature 460, 108-112 (2009).

41. Muranski, P. et al. Th17 cells are long lived and retain a stem cell-like molecular signature. Immunity 35, 972-985 (2011).

42. Maekawa, Y. et al. Notch controls the survival of memory CD4+ T cells by regulating glucose uptake. Nat. Med. 21, 55-61 (2015).

43. Pan, Y. et al. Survival of tissue-resident memory $T$ cells requires exogenous lipid uptake and metabolism. Nature 543, 252-256 (2017).

44. Reya, T. et al. A role for Wnt signalling in self-renewal of haematopoietic stem cells. Nature 423, 409-414 (2003).

45. Gattinoni, L. et al. Wnt signaling arrests effector T cell differentiation and generates CD8+ memory stem cells. Nat. Med. 15, 808-813 (2009).

46. lijima, N. \& Iwasaki, A. T cell memory. A local macrophage chemokine network sustains protective tissue-resident memory CD4 T cells. Science 346, 93-98 (2014).

47. Kondrack, R. M. et al. Interleukin 7 regulates the survival and generation of memory CD4 cells. J. Exp. Med. 198, 1797-1806 (2003).

48. Glennie, N. D., Volk, S. W. \& Scott, P. Skin-resident CD4+ T cells protect against Leishmania major by recruiting and activating inflammatory monocytes. PLoS Pathog. 13, e1006349 (2017).

49. Nakae, S. et al. Antigen-specific T cell sensitization is impaired in IL-17-deficient mice, causing suppression of allergic cellular and humoral responses. Immunity 17, 375-387 (2002).

50. Adachi, Y. et al. Distinct germinal center selection at local sites shapes memory B cell response to viral escape. J. Exp. Med. 212, 1709-1723 (2015). 